
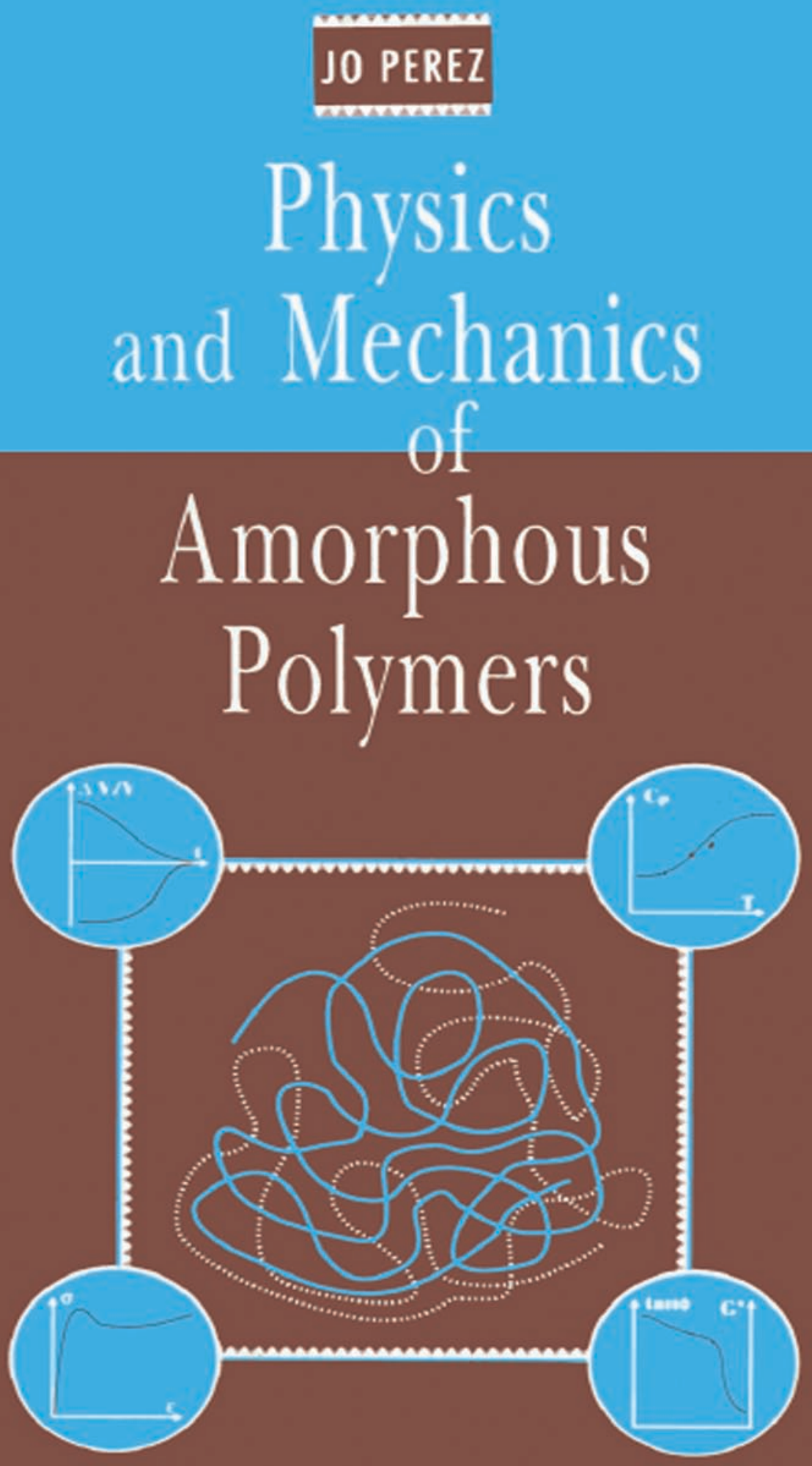
Physics and Mechanics of Amorphous Polymers 


\section{$\Rightarrow$ Taylor \& Francis Taylor \& Francis Group \\ http://taylorandfrancis.com}




\title{
Physics and Mechanics of Amorphous Polymers
}

\author{
Jo Perez \\ Professeur INSA \\ Lyon-Villeurbanne \\ France
}

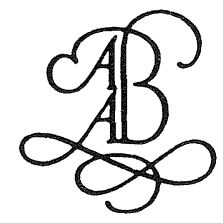

A.A.BALKEMA/ROTTERDAM/BROOKFIELD/I998 
Published by arrangement with Technique et Documentation Lavoisier, Paris

Published with the assistance of the Counsellor for Cultural, Scientific and Technical Cooperation, Embassy of France, New Delhi.

Translation of : PHYSIQUE ET MÉCANIQUE DES POLYMÈRES

AMORPHES. Technique et Documentation Lavoisier, 1992.

ISBN 2-85206-787-0

Published by

A.A. Balkema. P.O. Box 1675, 3000 BR Rotterdam, Netherlands

Fax: +31.10.4135947; E-mail; balkema@balkema.nl; Internet site: http://www.balkema.nl

Distributed in USA and Canada by

A.A. Balkema Publishers, Old Post Road, Brookfield, VT 05036-9704, USA

Fax: 802.276.3837; E-mail: info@ashgate.com

ISBN 9054107669

(1) 1998 A.A. Balkema, Rotterdam 


\section{Foreword}

The Physicist knows that change in internal structure is a central problem of his discipline, a problem that is both very old and very difficult. Changes of order have posed a real obstacle to understanding. The dynamics of the systems has, however, made substantial progress in the last few years: although is not totally resolved even for simple systems, the problem of changes of structure has been considerably clarified.

L'ordre et la volupté- $\mathrm{R}$. FIVAZ

The technology of polymers has been characterised since the 1980s as follows:

- at the level of industrial production, by large movements of rational restructuring at the world level. In Europe, the more or less dominant role is played by such groups as Atochem, BASF, Bayer, Hoechst, ICI, Rhône Poulenc and others.

- at the level of research and development, by a serious slowing down in the perspectives new chemical products seem to emerge and by further extension of efforts towards a better understanding, control and application of polymers already well established. Thus we observe a strong competition between thermosets and thermoplastics.

In the present economic environment, and considering the appearance of new producers (countries rich in petrol), it is indeed clear that research on polymeric materials is essentially oriented towards the economy of energy and of matter in the chemical chain formulation obtained and particularly towards control of product technics offering maximum value added. This is the case in particular of polymers with high glass transition temperature (Polyimides, PEEK, avimides etc.).

Under these conditions, we may schematically consider, among others, three domains in which the activity of research on polymeric materials gains obvious importance:

(a) the range of mechanical properties: resistance to fracture, tenacity, plasticity, flow, loss in cyclic loading, modulus of elasticity, anelasticity etc.;

(b) the range of methods of characterisation. To the more or less classical spectroscopic methods of local characterisation (IR, Raman, NMR, EPR, etc.) it is 
necessary to add the methods of semi-local or long distance characterisation such as X-ray (diffraction, scattering), electron microscopy (TEM, SEM), mechanical spectrometry etc. These methods of characterisation require acquisition of information at the microscopic scale (molecular level: conformational state, molecular orientation etc.) and at the macroscopic (morphology of monophase domains, spherulites etc.) by passing through the mesoscopic state (crystallites, nanoparticles etc.); all this information leads to better understanding, control and prediction of macroscopic behaviour;

(c) realisation of the most diverse morphologies depending on the thermal, mechanical or physicochemical conditions undergone by the polymeric materials. Efforts have also been made to develop blends (or 'alloys') of polymers representing the effects of synergism.

The nature of these three domains of research emphasises the current interest in the multidisciplinary approach for further progress in the knowledge of solid polymer, to begin with the simplest: the amorphous polymer solids. This is the theme of the present book.

This book is the result of a decade of research on amorphous polymers conducted in the GEMPPM Laboratory of INSA of Lyon (Group for Studies in Metallurgical Physics and Physics of Materials): i.e. it has been written in that spirit wherein this multidisciplinary character is of prime importance. Naturally this is not without danger: certainly, the specificity of the chemical nature of the systems discussed will not be well taken into account, among others, analysis of the law of mechanical behaviour will not be sufficiently rigorous and finally it will not miss the authorised opinion that without considering the Hamiltonian of interacting units constituting the amorphous polymers, the physical character of the state of matter envisaged here is far from convincing. Sometimes the systematic character of approach that led to the present work, gives rise to the development of original concepts (not in their nature: we find these concepts, or their equivalents, in the literature of this last decade, but rather in their application to the amorphous polymers). With the help of these concepts, it seems possible to have a unified picture of the very diverse phenomena shown by these materials, i.e. the phenomena among which the mechanical and micromechanical properties occupy a prominent status in this book. To understand the plasticity of amorphous polymers in a solid state, the increase in dynamic modulus with temperature, the flow, the experiments of relaxation of specific volume or of enthalpy and more generally the effect of physical aging on all these phenomena, in the light of those physical hypo.heses intervening the same set of parameters (with a reduced number of hypotheses and parameters), is an undisputably convincing objective. Does it suffice for the simplifications imposing inevitably on this type of approach to be considered valid and acceptable? Certainly, this is left to the judgment of the reader but, as is usual with the science of materials, experimental studies will referee! 
Seven chapters and two Appendices constitute this book. A 'List of Symbols' used in the book appears in the preliminary pages.

The last pages of this book enable, in conclusion, an assessment of the journey performed along these seven chapters and give rise to various consequences and applications to which a good knowledge of physical and mechanical aspects of amorphous polymers may lead.

Each chapter concludes with a heading 'For further study', wherein some bibliographic references (deliberately few) will enable the reader to obtain deeper knowledge of the 'key' points discussed in the chapter.

Let us mention finally that much of this book is devoted to numerical simulation: parallelly on the experimental side, the computer is a tool whose use cannot be neglected. We may consider theoretical modelling experiments an effective means of advancement in the domains of research concerning the physics of condensed matter.

Such a book must be accessible in terms of a good basic scientific knowledge; it is addressed to all those interested in the science of polymers, to teachers (2nd and 3rd cycle) as well as those engaged in research. However, it must be noted that it pertains more to scientific experiments than to work whose aim is exclusively pedagogic. Therefore, it proposes a journey through the domain of physics and mechanics of solid amorphous polymers, which the reader will probably find unique but the author hopes convincing as well. Naturally, such a journey is possible only when we accept the point of view so well illustrated by the following lines:

Experimentation per se is incapable of discovering the cause (or causes) of a phenomenon. In all cases it is necessary to extend the real by the imaginary and subsequently prove this halo of the imaginary which completes the real. This jump into the imaginary is fundamentally a 'mental' operation, a 'Gedenkenexperiment', for which no apparatus can exist. Claude Bernard saw this aspect very lucidly and in his scheme Observation-Idea-Experimentation, the psychological processes creating the idea (concept) are left in total obscurity, but he insists on their necessity [...]. In other words, the experiment to be scientifically significant, does not surpass the expectation. [...].

Conclusions: the experiment is guided either by an immediate technological need (for example: to test the properties of such or such materials under such and such condition) or by a hypothesis, the result of a mental experiment (Gedenkenexperiment) which precedes it and which we wish will be adequately close to the real. 


\section{$\Rightarrow$ Taylor \& Francis Taylor \& Francis Group \\ http://taylorandfrancis.com}




\section{List of Symbols}

$A_{0}$ :

$A_{1}$ :

$A_{a}:$

$A_{\beta}\left(A_{a n}\right):$

$a_{0}, a_{1}$ :

$a_{d}:$

$a_{T}, a_{8}:$

$a_{\tau}\left(a_{\jmath}, a_{G}\right):$

$\alpha:$

$\alpha_{l}\left(\alpha_{v}\right)$ :

$\Delta \alpha$ :

$\Delta \alpha_{v}\left(\Delta C_{p}, \Delta \chi\right):$

$b$ :

$<b>$ :

$B$ :

$B_{1}, B_{2}$ :

$B_{b}\left(B_{\chi}\right)$ :

$b_{\alpha}\left(b_{\beta}\right)$ :

$b_{a n}\left(b_{v p}\right):$ constant related to rate of viscoplastic deformation at stress and at characteristic time of this deformation

coefficient relating rate of viscoplastic deformation and creation of quasi-point defects

area of activation

intensity of $\beta$ relaxation (anelastic)

coefficients intervening in the relation between concentration of quasi-point defects, viscoplastic deformation and correlation parameter $\chi$

constant expressing degree of deformability of an atom (or group of atoms)

parameter of change in characteristic time from structural relation (Kovacs)

shift factor of curves of characteristic time, compliance, dynamic shear modulus in plots as function of $\log (t)$

constant of polarisation

coefficient of linear (or volumic) thermal expansion

difference in polarisation (between two directions)

difference between values of $\alpha_{v}\left(C_{p}, \chi\right)$ in supercooled liquid and glassy states

factor involved in Kohlrausch extended exponential; equivalent to correlation parameter during 'hierarchical series' of correlated movements and of purely Brownian origin $(0<b$ $<$ 1)

average value of shearing vector $\overrightarrow{\mathbf{v}}_{d}$

compressibility modulus

coefficients occurring in relation between structural relaxation time and molecular mobility during an experiment of plasticity

parameter of Gaussian distribution of $1 / b_{i}\left(1 / \chi_{i}\right)$

factor of distribution of characteristic times of relation $\alpha(\beta)$

parameter of Gaussian distribution of characteristic times of anelastic (viscoplastic) deformation 
$\beta$ :

parameter regulating non-exponentiality in the function of relaxation $F_{r}(t)$

C: curve

$C_{1}$ : constant characterising deformability of a structural unit (in $\mathrm{m}^{3} \mathrm{~J}^{-1}$ )

$C_{A G}\left(C_{A G a v}\right): \quad$ constant involved in the theory of Adam and Gibbs, of the dimension of a frequency (mean value)

$C_{\text {su }}$ : concentration of structural units in a solvent (number per unit of volume)

$C_{\text {attr }}$ and $C_{r e p}$ : constants occurring in the relation expressing the LennardJones potential

$C_{d}$ : concentration of quası-point defects

$C_{d}$ (stat):

$C_{E}$ :

$C_{\Delta U}:$

$C_{p}$ and $C_{v}$ :

$\mathrm{Cr}_{\mathrm{r}}$ :

$C_{b c}^{1}, C_{b c}^{2}:$

$C_{f v}$ :

$C_{s 1}, C_{s 2}$ value of concentration of quası-point defects established during stationary regimes of plastic deformation

constant occurring in the Eyring relation (dimension of rate of shearing)

factor characteristic of the distribution of two populations between two asymmetric wells

specific heat, specific volume

constant situating $T_{1}$ with respect to $T_{g}\left(C_{T}=T_{g}\left[T_{g}-T_{\mathrm{l}}\right]\right)$

coefficients appearing in the relation between $b$ and $C_{d}$

constant (between 0 and 1) involved in the theory of free volume developed by Cohen and Turnbull

constants involved in the relation between configurational entropy and concentration of quasi-point defects

$C_{W L F}(1), C_{W L F}(2)$ : coefficients of $W L F$ law (values termed universal; respectively 17.44 and $51.6 \mathrm{~K})$

$\chi_{c}: \quad$ compressibility $\left(\chi_{c}=1 / B\right)$

$\chi$ : correlation coefficient in the case of a system evolving under the effect of a stress

$D: \quad$ coefficient of diffusion; $D_{0}$ : constant of dimension of a coefficient of diffusion

$d$ : dimensionality

$\overrightarrow{\mathbf{d}}$ : direction taken by a linear defect

$\delta_{E}$ : $\quad$ parameter characterising the energy of cohesion l $\left(\mathrm{j} \mathrm{mol}^{-1} \mathrm{~m}^{-3}\right)^{1 / 2} \mathrm{~J}$

$\delta_{f}$ : $\quad$ number corresponding to a fractal dimension

$\delta(t)$ : $\quad$ relative deviation of volume with respect to equilibrium value

$\delta_{t a}$ : $\quad$ parameter translating the effect of thermoactivation in the theory of mode coupling

$E$ : tensile modulus (Young's modulus)

$E_{l}$ : $\quad$ energy of line of linear defect per molecular length 
$E_{a v}: \quad$ height of potential energy barrier limiting the migration of structural units (diffusion)

$\varepsilon$ : deformation (tensorial quantity $\varepsilon_{\alpha \beta}$ with $\alpha, \beta=1,2,3$; we often use $\varepsilon$ in place of $\varepsilon_{11}$ or $\varepsilon_{\text {shear, }}$ tensile or shear component of $\varepsilon$ ); we separate $\varepsilon$ into its elastic component $\varepsilon_{e l}$ and non-elastic component $\varepsilon_{n o n-e}$, the latter comprising anelastic $\varepsilon_{a n}$ and viscoplastic $\varepsilon_{v p}$ parts: $\varepsilon_{0}$ : dimensionless constant: $\Delta \varepsilon_{\beta}\left(\Delta \varepsilon_{a n}, \Delta \varepsilon_{p p}\right)$ : elementary local shearing related to the $\beta$ relaxation (anelasticity, the viscoplasticity)

$\varepsilon_{v}$ : $\quad$ permittivity of vacuum

$\Delta E_{1}$ : $\quad$ difference in energy levels between trans and gauche conformations

$\Delta E_{2}$; height of energy barrier separating two neighbouring conformations

$\Delta E_{a}\left(\Delta H_{a}, \Delta G_{a}, v_{a}\right)$ : energy (enthalpy, free enthalpy, volume) of activation

$\eta$ :

$F:$

viscosity: $\eta_{0}$ : constant of dimension of viscosity

$F_{m u}:$

force: $F_{u}\left(F_{t}\right)$ : applied (reaction) force

$F_{q}(t):$

molar attraction constant (same unit as $\delta_{E}$ )

$F_{r}(t):$

autocorrelation function

$\phi:$

relaxation function

$\Phi:$

dephasing angle between stress and deformation

$G\left(G_{l}, G_{r}, G_{r u b}\right)$

diameter of structural cages

shear modulus (instantaneous value, relaxed value, rubbery modulus)

$G_{G}:$

$\Delta G_{\beta}:$

$\overrightarrow{\mathbf{g}}_{i}$ :

H:

$\Delta H_{r}$ :

Gibbs free enthalpy; $\Delta G$ : variation of molar free enthalpy

intensity of $\beta$ relaxation (difference between value of real modulus before and after relaxation)

$\Delta H_{F}\left(\Delta S_{F}\right)$ : vector joining centre of gravity of chain and $i$ th unit

enthalpy

enthalpy of reaction of system on mobile entity

enthalpy (entropy) of formation of a quasi-point defect: the corresponding free enthalpy is thus $\Delta G_{F}=\Delta H_{F}-T \Delta S_{F}$ : with a distribution. we have $\Delta H_{F}(i)=\Delta H_{0}+i H_{1}$ and $\Delta S_{F}(i)=\Delta S_{0}+i S_{1}$ : in the case of monomers the notation is $\Delta h_{l}$ and $\Delta s_{l}$

$\Delta H(T), \Delta S(T)$ : increment in enthalpy, entropy of a disordered system with respect to the ordered state (function of temperature)

$h$ : Planck constant

$J(t)$ : compliance as a function of time; $J *(i \omega)$ : complex compliance: $J_{e} . J_{a n}, J_{p p}$ : components respectively elastic. anelastic and viscoplastic of compliance; $J_{t}$ and $J_{r u b}$ : instantaneous and rubbery compliance; $J_{r}$ : relaxed compliance with respect to any relaxation phenomenon (for $\alpha$ relaxation, $J_{r}=J_{\text {ruh }}$ ) 
$k$ :

$L:$

$L_{0}$ :

$L_{f}$ :

$l_{a v}$ :

$l_{\text {cor }}$ :

In, log:

$\lambda$ :

$\lambda_{t}$ :

$\Lambda:$

$M$ :

$m$ :

$\mu:$

$\mu_{r}$ :

$\mu_{z}, \mu^{0}$ :

$\Delta \mu:$

$N$ :

$N_{A}:$

$N_{d}:$

$N_{e}$ :

$n *$ :

$n_{\sigma}$ :

$N_{n}, \mu_{n}$ :

$n_{d l:}$

$N_{\text {tot: }}$ :

$N_{s s:}$

$n_{i}\left(n_{i l}, n_{i t}\right)$ :

$n_{h}:$

$v$ :

$v_{0}$ :
Boltzmann constant

length of a macroscopic object (for example: test specimen)

initial length

latent heat of fusion

mean distance between quasi-point defects

correlation length

neperian, decimal logarithm

dimension of a structural unit, precisely equal to the distance between two adjacent structural units

length of macromolecular chain having only 'trans' conformations

elongation $\left(\Lambda=L L_{0}\right)$

general notation for a modulus (shear: $G$; tensile: $E$ )

exponent of $\sigma$ in the power law relating $d \varepsilon / d t$ to $\sigma$

thermodynamic potential

shift factor of compliance- $\log (t)$ curve (experiment of aging)

parameters involved in the theory of hierarchical correlated movements

thermodynamic potential barrier to be crossed during a cooperative movement

number of repeat units in a chain

Avogadro number

number of structural units in the defect site amongst the total number $N_{A}$; thus we have $C_{d}=N_{d} / N_{A}$

number of subchains per unit volume

number of quasi-point defects where an smd might possibly be nucleated

coefficient of dependence of rate of deformation on stress

number of particles on the level $n$ in an Ising model $\left(\mu_{n}<\right.$ or $\left.=N_{n}\right)$

number of degrees of freedom

total number of sites considered in a macroscopic object; $N_{0}$ :

number of sites per unit volume $\left(N_{t o t}=N_{0} \times V_{t o t}\right)$

number of subsystems constituting the macroscopic system

indices of refraction (longitudinal and transverse value:

$\left.\Delta n_{i}=n_{i l}-n_{i t}\right)$

random number

critical exponent involved in the relation between $R_{f}$ and $N$ vibration frequency of a microphysical system in its fundamental state $\left(v_{0}=1 / \tau_{0}\right) ; v_{E}, v_{D}$ : frequency of Einstein, Debye $\left(\sim 10^{13} \mathrm{~Hz}\right) ; v_{f}$. frequency of excitation during a dynamic measurement: the angular frequency is $\omega=2 \pi v_{f}$ 


$v_{p}:$
$P:$
$p_{1}, p_{2}:$
$Q:$
$q_{1}$ and $q_{2}:$
$\overrightarrow{\mathbf{r}}:$
$R\left(R_{a v}, R_{0}\right):$
$R_{f}:$
$R_{g}:$
$R_{t m}:$
$R_{t o r}:$
$R_{P D}:$
$\vec{r}$
$\rho:$
$S:$
$\sigma:$
$S_{q}:$

$t_{0}:$

$t_{\text {ug: }}$

$T:$
Poisson constant

hydrostatic prussure

dipole moments

parameter involved in expression giving complex modulus

constants involved in relations characterising structural relaxation time (Cunat)

vector joining the ends of a chain

radius of a microscopic matter (mean value, critical value)

Flory's radius

radius of gyration

rigidity of a mechanical test machine

total rigidity of machine + specimen

Prigogine-Defay ratio

value joining the two extremes of a chain: more generally, $r$ is used for denoting a distance

density $\left(\delta \rho_{q}\right.$ : fluctuation of density corresponding to a wave vector $q)$

entropy (molar value); $s$ : entropy of a subsystem; $s^{*}$ : minimum configurational entropy so that a subsystem can change configurational state: $\Delta S_{m}$ : entropy of mixture

static structure factor

surface; $S_{u}$ : area of a surface

stress; tensorial quantity $\sigma_{\alpha \beta}$ with $\alpha, \beta=1,2,3$; we frequently find $\sigma$ in place of $\sigma_{11}$ or $\sigma_{\text {shear }}$ longitudinal component of shear of $\sigma ; T_{r}$ and $D_{e}$ are respectively the trace and the deviator of $\sigma_{\alpha \beta} ; \sigma_{i}$ : internal stress leading to effective stress $\sigma_{e f f}=\sigma-\sigma_{i} ; \sigma_{0}$ : constant of dimension of a stress: $\sigma_{m}$ : stress required for crossing barriers by mechanical effect only ( $T=$ $0 \mathrm{~K}) ; \sigma_{1}, \sigma_{p}$ and $\sigma_{t r} ;$ threshold stress of plasticity. plastic flow and transitory component $\left(\sigma_{1}=\sigma_{p}+\sigma_{t r}(\max )\right)$

parameter of dimensions of time: we have $0<t_{0}<\tau(1)$ with $t_{0}$ being as small as the population (like the effect of correlation) of the various levels of correlated movements is large

\section{aging time}

temperature: $T_{c r i t}$ : critical temperature of transition in the case of a phase transition: $T_{g}$ : glass transition temperature: $T_{0}$ (Kauzmann) or $T_{2}$ (Adam. Gibbs. Di Marzio): temperature at which enthalpy and entropy of configuration become zero; by analogy: $T_{0}^{*}$ is the temperature at which $v_{f a v}=0 ; T_{i}$ : fictitious temperature; $T_{F}$ : temperature of fusion: $T_{\alpha}\left(T_{\beta}\right)$ : temperature of $\alpha(\beta)$ relaxation observed at $1 \mathrm{~Hz} ; T_{1}$ : temperature at which all the curves $\tau_{\alpha}(T)$ intersect 
$\tau$ :

$\tau *:$

$\tau_{\text {entr: }}$

$\tau_{\max }\left(\tau_{1 \max }\right):$

$\tau_{m o l}$

$\tau_{R}:$

$\tau_{s r}:$

$\tau(t)\left(\tau_{1}(t)\right)$

$\tau_{t g}:$

$\theta:$

$\Theta:$

$\Theta_{\tau}:$

$U$ :

$\Delta U:$ characteristic time ( $\tau_{i}:$ particular value in a distribution)

characteristic time of structural relaxation appearing in the phenomenological theory of Narayanaswamy; $t_{c}$ : time for which all the $\tau_{\alpha}(T)$ curves intersect each other; $\tau_{\alpha}\left(\tau_{\beta}\right)$ : characteristic time of $\alpha(\beta)$ relaxation; $\tau_{\alpha a v}$ and $\tau_{\beta a v}$ : mean values; $\tau_{\text {trans: }}$ : characteristic time of a translational molecular movement, either by mode coupling or by thermoactivated collective movement; $\tau_{c v}$ : characteristic time of molecular movement resulting from coherent vibration; $\tau_{0}$ : time corresponding to frequency of limited activation; $\tau_{0}^{*}$ : same quantity but taking into account entropy of activation (example of $\beta$ relaxation: $\tau_{0}^{*}=\tau_{0} \cdot \exp \left(-\Delta S_{\beta} / k\right)$

characteristic time of the redistribution of macromolecular chains under an entropic effect

limiting value of $\tau(t)\left(\tau_{1}(t)\right)$ : characteristic time from which a system evolves in an ergodic manner

average duration of movement of a structural unit on a distance of the order of its dimension, under the effect of only thermal vibrations

characteristic time of movement of segments of chains in a glassy medium (Rouse model)

lifetime of a configurational state; cquivalent to the characteristic time of structural relaxation

characteristic time of movements corresponding to the excitation of degrees of freedom involved in this movement at the end of the time $t$ after application of the stimulus 'variation of temperature' ('variation of stress')

characteristic time of establishment of thermodynamic equilibrium between various conformations

internal variable

angle between a chain segment and a reference direction; more generally, angle characterising the arrangement of matter

parameter of temperature dependence of structural relaxation time (Kovacs: relation between $a_{T}, a_{\delta}$ and $T$ )

energy of interaction $(U(r)$ : function of spatial coordinate; $U\left(r_{i j}\right)$ : energy of interaction of a pair of structural units $i$ and $j$, situated at a distance $r_{i j}$ ); $U_{\beta}$ : height of energy barrier opposing the elementary movement of the macromolecules difference in levels of energy between two adjacent wells in the configurational space 
$v:$

$V$ :

$v_{a}:$

$v_{\beta}:$

$\overrightarrow{v_{d}}$

$V_{s}:$

$v_{0}\left(v_{i}, v_{a v}\right)$;

$v_{1}$ :

$v_{f}:$

$v(T)$ :

$V_{\text {tot: }}$

$\Omega:$

$\vec{\omega}$ :

$x$ :

$\xi$

$y$ :

$z$

Z:

velocity; $v_{l}$ : velocity of mobile part of the mechanical test machine

molar volume

activation volume

coupling constant between stress and molecular configuration $\left(\mathrm{m}^{3}\right)$

shear vector associated with a linear defect ( $\Delta v_{d}$ : equivalent quantity corresponding to microloops of dislocation) specific volume

volume of structural unit (of unit $i$, of mean value) volume of matter concerned with elementary shear $\left(v_{f a v}\right)$ : free volume (mean value) parameter expressing excluded volume volume of a macroscopic object (test specimen) number of configurations axis of rotation parkmeter regulating non-linearity in the expression giving $\tau^{*}$ coefficient of molecular friction ( $\xi_{1}$ : monomeric coefficient) fraction of number of repeat units on which cross-linking occurs

number of structural units participating in a co-operative movement (minimum value: $z^{*}$ )

partition function 


\section{$\Rightarrow$ Taylor \& Francis Taylor \& Francis Group \\ http://taylorandfrancis.com}




\section{Contents}

Foreword $\mathbf{v}$

$\begin{array}{ll}\text { List of Symbols } & \text { ix }\end{array}$

Chapter 1: Structural Aspect of Polymers 1

1. Structure of Macromolecules 1

1.I Interatomic Bonds

1.2 Configurations in Macromolecular Chains 4

1.3 Conformation of Macromolecules 5

2. Polymers in Solution 7

2.1 Ideal Chain 7

2.2 Unperturbed Real Chain $\quad 8$

2.3 Real Chain Perturbed by Excluded Volume Effects $\quad 8$

3. Polymers in Molten State and in Solid State 10

3.1 Arrangement of Macromolecules 10

3.2 Intermolecular Interaction: Cohesion in Solid Polymers 12

3.3 Concept of Physical Defects in Solid Amorphous

Polymers 14

3.3.1 Concept of point defects 15

3.3.2 Linear defects 24

4. Conclusion 29

Further Reading $\quad 32$

Book $\quad 32$

Articles $\quad 32$

Theses $\quad 32$

Chapter 2: Molecular Mobility in Amorphous Solid Polymers 33

1. Introduction 33

2. Molecular Mobility in Supercooled Liquids 36

2.1 Free Volume Theory 37

2.2 Fluctuations of Entropy 41

2.3 Comparison of the two Theories: Discussion 43

3. Molecular Mobility in Glassy Solids 46

3.1 Configurational State of Glasses 46 
xviii Physics and Mechanics of Amorphous Polymers

3.2 Molecular Mobility: from Liquid to Glassy Solid $\quad 50$

3.3 Concept of Defect and Molecular Mobility 51

3.4 Molecular Mobility and Hierarchical Correlation Effects 54

3.5 Discussion and Comparison of Various Models $\quad 62$

4. Amorphous Polymers and Molecular Diffusion 64

5. Discussion and Conclusion 68

5.1 Local Molecular Motions $\quad 68$

5.2 Glass Transition $\quad 71$

$\begin{array}{ll}\text { Further Reading } & 74\end{array}$

$\begin{array}{ll}\text { Books } & 74\end{array}$

Articles $\quad 74$

$\begin{array}{ll}\text { Thesis } & 75\end{array}$

Chapter 3: Non-elastic Deformation of Solid Amorphous Polymers $\quad 76$

1. General Aspects $\quad 77$

2. Experimental Results $\quad 84$

2.1 Large Deformations $\quad 84$

2.1.1 Plastic deformation and molecular orientation 9]

2.1.2 Law of behaviour $\quad 92$

2.2 Small Deformations: Mechanical Spectroscopy,

Micromechanical Tests 95

2.1.1 Microflow $\quad 95$

2.2.2 Mechanical spectroscopy 96

2.2.3 Stress relaxation $\quad 101$

2.2.4 Thermostimulated creep (TSC) 104

3. Molecular Aspects 106

3.1 Plastic Deformation and Microstructural Change 107

3.2 Plastic Deformation and Internal Stress 109

$\begin{array}{ll}3.3 \text { Nature of Barriers } & 109\end{array}$

Further Reading $\quad 113$

$\begin{array}{ll}\text { Books } & 113\end{array}$

Articles 113

Theses 114

Chapter 4: Theoretical Approach of Non-elastic Deformation of Solid Amorphous Polymers $\quad 115$

1. Introduction: General Hypotheses 115

2. Theoretical Analysis $\quad 117$

$2.1 \beta$ Relaxation $\quad 117$

2.2 Sheared Microdomains $\quad 120$

2.3 Calculation of Deformation 123

2.3.1 Case of high temperatures $\quad 123$ 
2.3.2 Case of low temperatures $\quad 130$

Further Reading $\quad 137$

Books $\quad 137$

Articles $\quad 138$

$\begin{array}{ll}\text { Theses } & 138\end{array}$

Chapter 5: Mechanical Experiments: Interpretation of Results 139

$\begin{array}{ll}\text { 1. Flow } & 139\end{array}$

1.1 High Temperature and Large Deformation 140

1.2 High Stress and Low Temperature 140

1.3 Small Deformation and Low Temperature 143

$\begin{array}{ll}1.4 \text { Recovery of Deformation } & 147\end{array}$

2. Applied Rate of Deformation

(Compression, Shear and Tensile Tests) 149

2.1 General Considerations 149

2.2 Numerical Simulation $\quad 153$

2.3 Comparison with Thermodynamic Analysis of Plasticity 159

2.4 Conclusion $\quad 162$

3. Stress Relaxation 163

4. Mechanical Spectrometry 170

4.1 Expression of frequential Compliance $\quad 170$

4.2 Entropic Elasticity and Chain Flow 171

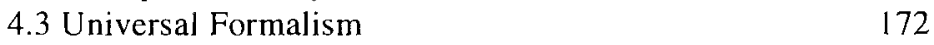

4.4 Application to Amorphous Polymers $\quad 175$

$\begin{array}{ll}4.4 .1 \beta \text { relaxation } & 176\end{array}$

4.4.2 $\alpha$ relaxation: isothermal behaviour

(metastable equilibrium) $\quad 179$

$4.4 .3 \alpha$ relaxation: behaviour at variable temperature $\quad 184$

$\begin{array}{ll}4.5 \text { Conclusion } & 186\end{array}$

5. Thermostimulated Creep $\quad 188$

5.1 Distribution of Characteristic Times 190

5.2 Phenomenon of Compensation 191

5.3 Discussion and Conclusions 194

6. Special Aspects of Mechanical Behaviour of Amorphous

$\begin{array}{ll}\text { Polymers } & 196\end{array}$

6.1 Peak of Stress 196

6.2 Kohlrausch Effect 200

6.3 Plastic Deformation and Dynamic Modulus 201

FURTHER READING 204

Articles $\quad 204$

$\begin{array}{ll}\text { Theses } & 205\end{array}$ 
Chapter 6: Physical Aging of Amorphous Polymers

1. Experimental Data 206

1.1 Mechanical Properties 207

1.2 Thermodynamic Properties $\quad 212$

1.3 Other Properties $\quad 214$

1.4 Conclusion 214

2. Thermodynamic Analysis 215

2.1 Thermodynamic Aspect 215

2.2 Phenomenological Theories 219

2.3 Thermodynamics of Irreversible Processes 223

3. Physical Analysis of Structural Relaxation 227

3.1 General Presentation 227

3.2 Molecular Aspects of Physical Aging 229

4. Numerical Simulation 234

5. Discussion and Conclusion 239

Further Reading 242

Book 242

Articles $\quad 242$

Theses $\quad 242$

Chapter 7: Glass Transition $\quad 243$

1. Experimental Aspect 245

2. Theories of Glass Transition 25I

2.1 Free Volume Theory 252

2.2 Theories Involving the Concept of 'Thermodynamic

Frustration' 252

2.3 Glass Transition and Phenomenon of Percolation 255

2.4 Molecular Dynamics and Glass Transition 256

3. Physical Bases for a New Approach to Glass Transition 257

3.1 Recapitulation of Concepts 257

3.2 Nature of Glass Transition 259

3.3. Measurement of $T_{g}$ : Comparison of Theories and
Principles of Main Experimental Methods

3.3.1 Calorimetric measurements 264

3.3.2 Dilatometric measurements 267

3.3.3 Thermomechanical properties 269

4. Discussion and Conclusion 273

4.1 Entropy of Glassy Systems: Kauzmann's Paradox 274

4.2 Glassy State and Hypersurface of Configuration 275

4.3 Glass Transition and Phenomenon of Percolation 277

4.4 Molecular Dynamics, Mode Coupling and Glass
Transition 
Further Reading $\quad 285$

Books 285

Articles $\quad 285$

Theses 285

Conclusion: Should there be one? 286

1. Summary of the Fundamental Points 286

2. Concepts to Accept (?) or Reject 289

2.1 Free Volume 289

2.2 VFT Law. WLF Treatment and Kauzmann Paradox 290

2.3 Principal $\alpha$ Relaxation and Glass Transition 291

2.4 True Modulus and Experimental Modulus 292

2.5 Glass Transition and the Theories of Mode Coupling 292

3. Problems and Scope of Extension 293

Appendixes $\quad 299$

A 1: Simulation of Liquid-Glass Cooling: Calculation of Properties from Thermal History 300

A2: Simulation of the Response (Deformation) for Various Mechanical Applied Stresses 


\section{$\Rightarrow$ Taylor \& Francis Taylor \& Francis Group \\ http://taylorandfrancis.com}




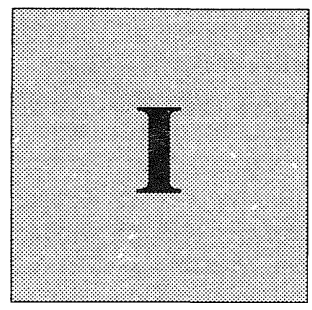

\section{Structural Aspect of Polymers}

\section{STRUCTURE OF MACROMOLECULES}

A macromolecule is formed by the repetition of a group of atoms termed a 'structural unit' or a 'repeat unit'. For example, the simplest macromolecule is polyethylene: the repeat unit is $\left[-\mathrm{CH}_{2}-\mathrm{CH}_{2}-\right]$.

For a given chemical composition, a macromolecule may have several configurations and several conformations.

Configuration describes the manner in which the atoms are bonded in the repeat unit: it corresponds therefore to those bonds which cannot be modified without irreversible transformation. Configurational isomers have the same composition but differ in arrangement of the bonds between atoms.

Conformation describes the arrangement of repeat units in the macromolecule. This arrangement may be modified in a reversible manner by rotation around the bonds between the atoms of the main chain of the macromolecule.

In this first section we describe the different types of bonds occurring between the atoms constituting a macromolecule; then we give essential information regarding the problems of configuration and thereafter consider the conformational variations in macromolecular systems.

\subsection{Interatomic Bonds}

It is well known that the covalent bond plays the principal role in the formation of macromolecules. This bond is of quantum nature and a direct consequence of the combination of electronic orbitals. Since the main atom in organic macromolecules is carbon, it is necessary to consider the orbitals in which the valency electrons of this atom are found. These orbitals present the phenomenon of hybridisation; the most common is the $s p^{3}$ hybridisation, for which the orbital $s$ and the orbital $p$ combine to form a hybrid orbital consisting of four lobes symmetrically arranged in the form of a tetrahedron. The molecule of methane is the most typical example of atomic configuration whose geometry arises from the 


\section{Physics and Mechanics of Amorphous Polymers}

directions of the covalent bonds between $\mathrm{C}$ and $\mathrm{H}$ (bonds constituted by the combination between the orbital $s$ of each $\mathrm{H}$ with a lobe of the hybrid orbital of $\mathrm{C}$ ).

Some values of energy of covalent bonds and the distances between concerned atoms are presented in Table I.1.

Table I.1

\begin{tabular}{ccc}
\hline Bond & Energy $(\mathrm{kJ} /$ mole $)$ & Distance $(\AA)$ \\
\hline$-\mathrm{C}-\mathrm{S}-$ & 280 & 1.81 \\
$-\mathrm{C}-\mathrm{C}-$ & 334 & 154 \\
$-\mathrm{C}-\mathrm{O}-$ & 355 & 1.46 \\
$-\mathrm{C}-\mathrm{H}-$ & 410 & 1.10 \\
$-\mathrm{N}-\mathrm{H}-$ & 418 & 1.02 \\
$-\mathrm{O}-\mathrm{H}-$ & 462 & 0.95 \\
$-\mathrm{C}=\mathrm{C}-$ & 612 & 1.34 \\
$-\mathrm{C} \equiv \mathrm{C}-$ & 836 & 120 \\
\hline
\end{tabular}

Among the other strong bonds occurring in macromolecular materials, let us also mention the semi-polar bonds (or co-ordinate bonds), for example the nitro groups:

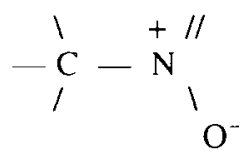

The bond between carbon and nitrogen is assured by ordinary covalence (one of the five electrons of nitrogen); two other electrons couple with two electrons of one of the oxygens (combination of one double orbital), thereby leaving a free doublet on nitrogen, which is shared with the other oxygen. Under these conditions the nitrogen is charged positively and the second oxygen negatively.

Finally. let us mention ionic interactions. Indeed, certain macromolecules carry ionisable groups, for example the acidic group:<smiles>CC(=O)[O-]</smiles>

Such electrically charged radicals may be bonded with ions (metallic cations: $\left.\mathrm{Na}^{+} \cdot \mathrm{Ca}^{++}, \mathrm{Mg}^{++}, \ldots\right)$.

Weak bonds are described less frequently; yet these bonds sometimes play an important role in the case of organic polymers. 
In general, weak bonds give rise to electrostatic forces which involve the following physical effects.

(a) Interaction between dipoles: Certain groups of atoms may give rise to a nonuniform distribution of electronic charges, implying that this group of atoms produces an electric dipole moment. Such dipoles possess an energy of interaction as given by Keesom's formula:

$$
U=-\left[\frac{1}{4 \pi \varepsilon_{v}}\right]^{2} \frac{2}{3 k T} \frac{P_{1}^{2} P_{2}^{2}}{\lambda^{6}},
$$

where $P_{1}$ and $P_{2}$ are the dipole moments and $\lambda$ the distance between them.

For a radius of interaction of 4 to $5 \AA$, we have an energy of dissociation of 10 to $15 \mathrm{~kJ} / \mathrm{mole}$.

The most classical examples of groups of atoms having a dipole moment are $=\mathrm{C}=\mathrm{O},-\mathrm{C} \equiv \mathrm{N}, \mathrm{C}-\mathrm{CI} \ldots$

(b) Effect of polarisation: A strong dipole may polarise a molecule or a group of atoms situated in close proximity, thus inducing a secondary dipole. This leads to the preceding case with an energy of interaction given by (Debye):

$$
U=-\left[\frac{1}{4 \pi \varepsilon_{v}}\right]^{2} \frac{4 \alpha P_{1}^{2}}{\lambda^{6}},
$$

$\alpha$ being the constant of polarisation.

The resultant forces of interaction are 5 to 10 times weaker than the preceding ones and have a radius of action from 3 to $4 \AA$. The ethylene double bonds and especially the aromatic rings are polarisable: these forces could therefore occur in the case of certain polymers.

(c) Dispersion forces: Even in the case of non-polar structural units (for example, an atom of rare gas), interaction occurs at every instant between the various electronic charges; over the course of time the average value of interaction energy is not zero even if the average value of the dipole moment is zero. London calculated the energy of interaction based on purely quantum mechanical considerations.

$$
U=-\frac{h v_{0} a_{d}^{2}}{2 \lambda^{6}}
$$

Here $a_{d}$ is a constant expressing the degree of deformability of the atoms (or groups of atoms).

Thus the so-called Van der Waals bonds comprise these three types of effects with varying relative magnitudes depending on the systems. In practice, this leads to an overall description of the interactions, which include 
the effect of repulsion by a single relation. The most widely used is that of Lennard-Jones whose second term corresponds to the various relations given above:

$$
U=\frac{C_{r e p}}{\lambda^{12}}-\frac{C_{a t t}}{\lambda^{6}} .
$$

The hydrogen bond, though generally stronger than the Van der Waals bond, is of the same nature, merely displaying a greater predominance of electrostatic and dispersive forces. This bond is responsible for the phenomenon of polymerisation in water and hydrofluoric acid, formation of ice, conformation of biological macromolecules etc. It also plays a significant role in the case of certain polymers, including polyamides as a typical example. Among the groups of atoms participating in the hydrogen bonds, the following are distinguished:

- the proton donor groups (acidic or alcohol group ...),

- the proton acceptor groups (ether group ...).

\subsection{Configurations in Macromolecular Chains}

We have already seen above that the simplest repeat unit is $\left[-\mathrm{CH}_{2}-\mathrm{CH}_{2}-\right]$; the replacement of one $H$ by another structural unit leads to the formation of vinyl chains. Several possibilities may be envisaged. The first relates to the succession of the repeat units:

- head to tail:

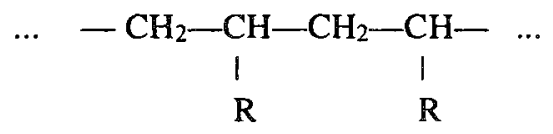

- head to head:

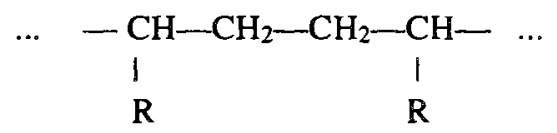

- tail to tail:

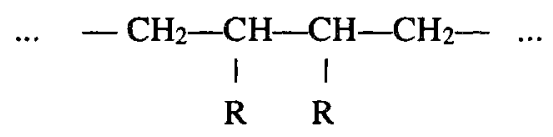

The second is relative to the existence of isomers from structural units having a double bond; the possibility of rotation around the axis joining the concerned carbons then does not occur. This results in isomers of different geometry. The classical example is that of polyisoprene:

\begin{tabular}{|c|c|c|c|c|c|}
\hline & $\mathrm{CH}_{3}$ & $\mathrm{H}$ & $\mathrm{CH}_{3}$ & $\mathrm{CH}_{2}$ & \\
\hline & 1 & I & 1 & I & \\
\hline Cis & $\mathrm{C}=$ & $\mathrm{C}$ & $\mathrm{C}=$ & $\mathrm{C}$ & Trans \\
\hline $\begin{array}{l}\text { (natural } \\
\text { rubber) }\end{array}$ & $\begin{array}{c}/ \\
-\mathrm{CH}_{2}\end{array}$ & $\begin{array}{l}1 \\
\mathrm{CH}_{2}-\end{array}$ & $\begin{array}{c}1 \\
-\mathrm{CH}_{2}\end{array}$ & $\begin{array}{l}1 \\
H\end{array}$ & (gutta perch \\
\hline
\end{tabular}


Finally, we must consider the tacticity: polymers may be isotactic (the structural units $\mathrm{R}$ are always found on the same side of the chain), syndiotactic (structural units alternate regularly on both sides of the chain) and atactic (structural units randomly distributed on one or the other side of the chain). As a general rule, it may be said that the tendency to crystallise and the type of crystalline ordering depend on the configuration of the repeat unit; thus the amorphous polymers described herein are often atactic.

\subsection{Conformation of Macromolecules}

Let us recall that the hybrid orbitals around carbon enable us to describe a sequence of these atoms bonded by covalent bonds such as a tetrahedral sequence bonded by its peaks. Figure 1.1 shows the simplest case, that of the 'ethane' molecule. That a rotational degree of freedom exists between one tetrahedron with respect to the other is very clear. However, all the positions are not equally probable: Figure I.1(c) indicates the variation of energy of this molecule with the angle of rotation $\theta$. The effects of interactions between hydrogens are minimised for $\theta=60,180$ and $300^{\circ}$, these three positions being equally probable.

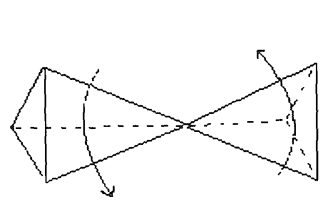

(a)

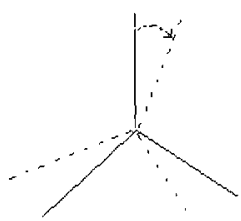

(b)

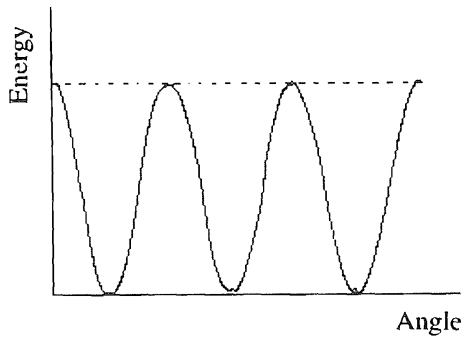

(c)

Fig. I.1. Rotational degrees of freedom of 'ethane' molecule and energy profile.

The situation is different for the 'butane' molecule. Figure I.2 shows that only one stable position exists: $\theta=180^{\circ}$; the other two minima $\left(\theta=60^{\circ}\right.$ and $\left.300^{\circ}\right)$ correspond to metastable states. The conformation is called trans in the first case and respectively gauche $(+)$ and gauche $(-)$ in the other two. The same situation is found in the sequence $i, i+3$ of 4 successive carbons of a macromolecular chain. Figure I.2(c) indicates the possibility of rotation of carbon $i+4$ and Figure I.2(b) variation of the energy of this sequence as a function of $\theta$. It is necessary to distinguish the difference in level of energy $\Delta E_{1}$ and height of the barrier $\Delta E_{2}$ to be jumped in order to pass from trans to gauche conformations; $\Delta E_{1}$ conditions the statistical distribution of conformations along the chain. Thus one can calculate the average length of segments of the chain having only trans conformations: 


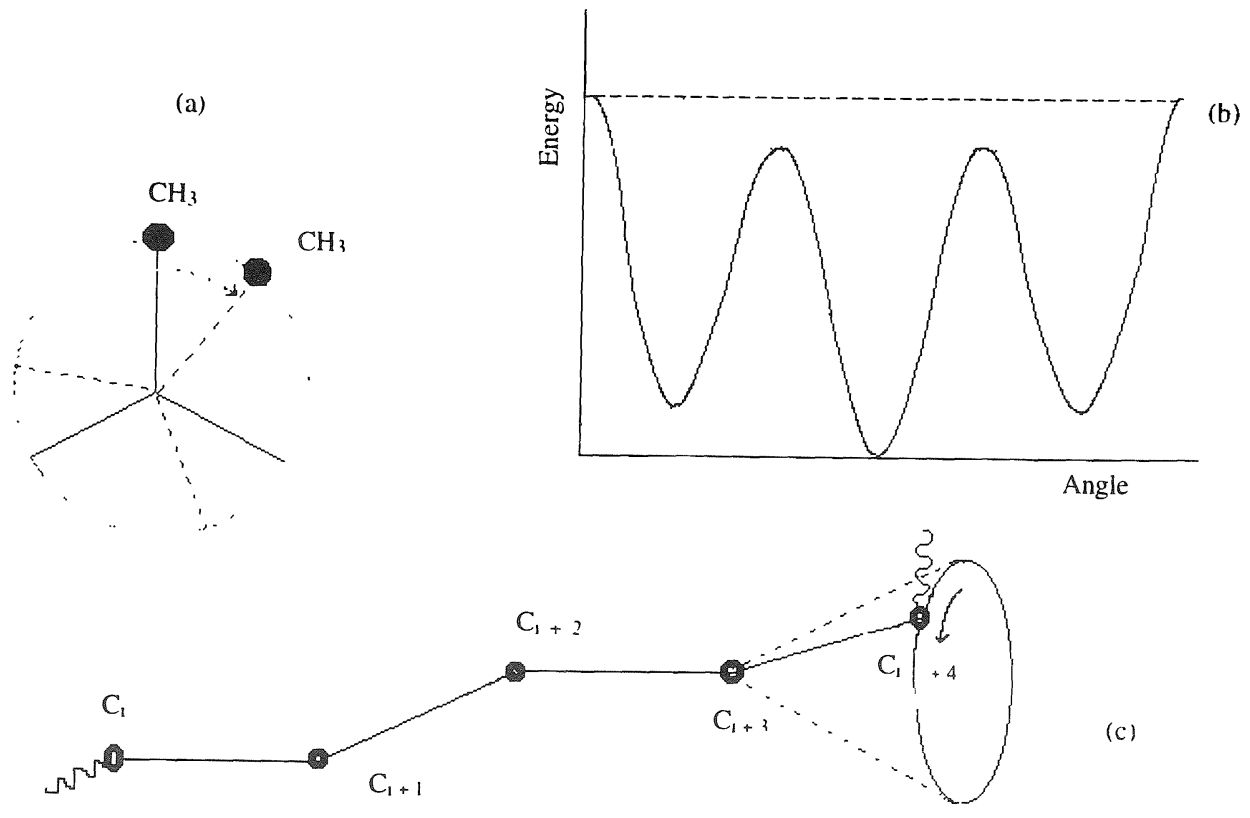

Fig. I.2. (a) and (b): Rotational degrees of freedom and energy profile of 'butane' molecule: (c) the same energy profile corresponding to each series of 4 carbons in a carbon chain macromolecule.

$$
\lambda_{t}=\lambda\left[1+0.5 \exp \left(\Delta E_{1} / k T\right)\right] .
$$

It is also necessary to take into account the time required to attain statistical equilibrium distribution. Hence a characteristic time $\tau_{t g}$ which depends, naturally, on $\Delta E_{2}$ is introduced:

$$
\tau_{i g}=\tau_{0} \exp \left(\Delta E_{2} / k T\right) .
$$

This leads to a distinction between rigid chains $\left(\Delta E_{1}>k T\right)$ and flexible chains $\left(\Delta E_{1} \leq k T\right)$. According to the value of $\tau_{t g}$, we may have dynamic flexibility $\left(\Delta E_{2} \leq k T\right)$ or, contrarily, a gel of unbalanced conformation $\left(\Delta E_{2} \gg k T\right)$.

A statistical thermodynamic analysis of macromolecular chains has been developed which enables calculation of conformational potential energy. By this technic the various types of conformation of macromolecules existing in a crystalline state are explained, in particular the following:

- Fully extended conformations: all the segments exhibit trans conformation (example; crystallites of polyethylene).

- Helical conformations, generally induced by the presence of complex structural units for which the extended chain would exert too high stresses on the bonds (example: crystallites of polypropylene). 
In the case of amorphous polymers the same statistical thermodynamic approach provides important information about the structural aspects of the macromolecular arrangements. In the next section we shall consider the case of polymers in dilute solution, a situation simplified by the fact that the intermolecular interactions can be neglected. In Section 3 we shall discuss the case of molten or solid polymers.

\section{POLYMERS IN SOLUTION}

Although the objective of this book pertains to solid polymers, it is worthwhile to recall the facts established for polymers in solution.

In view of the complexity of the study of real chains and the sufficiently high degree of polymerisation, conformational statistics of simplified models have been developed. We shall thus first describe an ideal chain model, the model of an unperturbed real chain and, finally, the model of real chains perturbed by excluded volume effects.

\subsection{Ideal Chain}

The structure of the chain (irrespective of the scale considered) is reduced to the sequence of $N$ links of length $\lambda$, with no orientational correlation. Restrictions on the angles between two successive links, in particular the existence of unfavourable positions, are ignored. The links have negligible volume and could therefore cross each other or occupy the same position in space.

Such a chain is thus considered a succession of $N$ vectors of equal length but in any direction. The vector $\mathbf{r}$ joining the two ends is the sum of these $N$ vectors.

With the assumption of random distribution of spatial orientations, the distribution function of end-to-end distances can be calculated. A Gaussian function is obtained from which it is possible to derive the free energy and thus the entropy of this ideal chain as a function of elongation $r$ :

$$
S(r)=S(0)-\frac{3 r^{2}}{2 N \lambda^{2}} .
$$

The entropy of the chain is maximum for a distance $r$ zero. This suggests that the chain in its equilibrium position is folded on itself. The conformation is called 'coiled'.

This approach enables us to also define:

- the mean square of $r:\left\langle r^{2}\right\rangle=N \lambda^{2}$,

- the radius of gyration $R_{g}=\left\langle g^{2}\right\rangle^{1 / 2}$ with

$$
\left\langle g^{2}\right\rangle=1 / N \sum_{i=1}^{N} g_{i}^{2}=N \lambda^{2} / 6,
$$

where $g_{i}$ denotes the vector joining the centre of gravity of the macromolecule to the $i$ th unit of the chain. 
Sometimes $\left\langle r^{2}\right\rangle^{1 / 2}$ is called Flory's radius.

In summary, the ideal chain model leads to the values:

$$
\begin{gathered}
R_{F}^{i}=N^{1 / 2} \lambda ; \\
R_{g}^{i}=(N / 6)^{1 / 2} \lambda .
\end{gathered}
$$

\subsection{Unperturbed Real Chain}

In this model the orientation of segments is no longer random since the values of the bond angles and the steric hindrances of rotation about the bonds are taken into account.

Gaussian statistics is still valid in this case but the statistical unit has changed: here we consider a sequence of segments of length $\lambda_{t}$ having only trans conformations. The number of these segments is $N_{t}$ and we have $N_{t} \cdot \lambda_{t}=N \cdot \lambda$.

The model of the unperturbed real chain thus gives:

$$
\begin{gathered}
R_{F}^{r}=N^{1 / 2}\left(\lambda \cdot \lambda_{t}\right)^{1 / 2} ; \\
R_{g}^{r}=(N / 6)^{1 / 2}\left(\lambda \cdot \lambda_{l}\right)^{1 / 2} .
\end{gathered}
$$

The conclusion of these results is that the value of Flory's radius and the radius of gyration increase in the same proportion as the chain becomes more and more rigid. For flexible chains where $\lambda_{t} \sim \lambda$, we obtain the aforesaid results. But in all cases the radii vary as $N^{1 / 2}$. Now all the experimental results (light scattering, viscosity measurements etc.) indicate rather a variation in proportion to $N^{0.6}$ This is explained in the next section.

\subsection{Real Chain Perturbed by Excluded Volume Effects}

The segment of a real chain in dilute solution in a good solvent have a certain volume and overlap of the chain segments is not possible. Only the conformations for which no pair of structural units occupies the same position in space are possible. The excluded volume effect consequently decreases the number of equilibrium conformations of the macromolecular coil, thus increasing the average dimensions of this coil.

We shall now summarise the method used by Flory for describing a macromolecular coil in a good solvent. By 'good solvent' we mean one whose molecules tend to surround the structural units of the macromolecular chain. In other words, the effect of interaction between solvent molecule and polymer structural unit is more attractive than the effect of structural unit-structural unit interaction. This difference in energy of interaction is used in the calculations of Flory.

Let $R$ be the radius of a macromolecule in such a solvent. The volume concentration of structural units is close to $c \sim N / R^{d}, d$ being the dimensionality. The repulsive energy (in the direction described above) is proportional to the 
number of bonds between structural units, i.e., proportional to $c^{2}$. Flory suggests (per unit volume):

$$
U_{\text {repul }} \sim T v(T) c^{2},
$$

where $v(T)$ is a parameter expressing the excluded volume, i.e., the fact that the repeat units are surrounded by solvent molecules, the volume of the coil increases.

For the volume of the macromolecule, we have:

$$
U_{\text {repultot }} \sim T v(T) c^{2} R^{d}=T v(T) N^{2} / R^{d} .
$$

On the other hand, the Gaussian statistical analysis gives us the elastic energy (rubber elasticity):

$$
U_{\text {elast }}=T R^{2} / N \lambda^{2}
$$

By minimising the total energy $U_{t o t}=U_{e p u l t o t}+U_{e l a s t}$, we obtain an expression of Flory's radius:

$$
R_{F}=d / 2 v(T) \lambda^{2} N^{v}
$$

with

$$
v=3 /(d+2) \text {. }
$$

We may thus state:

- With dimensionality $d=3, v=3 / 5$, i.e., $R_{F} \sim N^{0.6}$, so the results accord well with experimental data;

- For $d=4$, we obtain the result as derived in the case of ideal chains;

- Such a result presumes $v(T)$ positive, implying that the segment-solvent interactions are predominant and the macromolecular chain has a tendency to surround itself by the molecules of the solvent (in other words, the effect of repulsion between segments). In a bad solvent, contrarily, the segment-segment interaction is attractive and the chain tends to fold over itself by 'expelling' the molecules of solvent that surround it. We may say that the effects of excluded volume are 'neglected'. This situation may occur in any solvent, either by varying the concentration of polymer in the solvent, or by varying the temperature. In the latter case, at a characteristic temperature termed $\theta$, the contraction can compensate the dilution due to the effects of excluded volume; the chain then behaves in an ideal manner.

Now, we know that Flory's approach is very approximate but the overestimation of repulsive energy is counterbalanced by the underestimation of elastic energy. Furthermore, the conclusions of this approach have been well confirmed by experimental observations. Actually the group theory of renormalisation allows justification of the value of various critical exponents, notably the exponent $v \approx 0.6$.

To summarise, the polymers in dilute solution in a good solvent exist in the form of isolated macromolecular coils, showing the behaviour ' of the excluded volume effect between structural units. 
What happens when the concentration of polymer in a solvent is increased? With a critical concentration $c^{*}$, the coils come into contact and with higher concentrations, the polymer chains begin to interpenetrate. Strong interactions of the type entanglement of different chain segments modify the behaviour described above. Schematically stated, the picture of the polymer solution is that of a network formed of domains of dimensions $l_{c o r}$ (denoted as correlation length) well described by the excluded volume model. surrounded by the matter formed from the various entangled macromolecules, in which the excluded volume effects are neglected.

When the concentration is increased, $l_{c o r}$ decreases and becomes zero for a critical concentration $c^{* *}$. The excluded volume effects are then negligible at all scales. Figure I.3 summarised the various cases. Given this information, we can now consider the situation of polymers first in a molten state and then in a solid state.

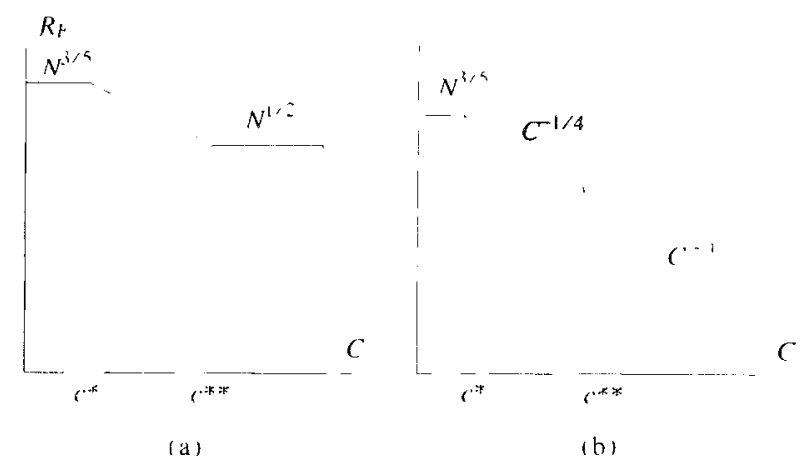

Fig. 1.3. Variation of Flory's radius (a) and dimension of the subchain (b) with concentration

\section{POLYMERS IN MOLTEN STATE AND IN SOLID STATE}

We have just seen that for a concentration tending towards 1 , the behaviour of macromolecules appears to be that of ideal chains: thus $R_{F}$ varies as $N^{1 / 2}$. Sometimes the Gaussian chain assumption is not valid, considering the strong interactions between the neighbouring structural units. This was shown by Flory and De Gennes. We shall describe in this section how the macromolecules arrange themselves with respect to each other. We shall then emphasise the importance of intermolecular interactions in relation to the concept of cohesion and elastic modulus. We shall finally see how the concept of 'physical defects' can be introduced on the basis of these preceding aspects.

\subsection{Arrangement of Macromolecules}

Let us consider a succession of macromolecular chains of the same nature in the state of condensed matter. Let us denote by $U$ the potential interaction between a 
chain $i$ and chains $j \neq i$. $U$ is essentially proportional to the local concentration $c$ of repeat units but $c$ has iwo components: one relative to chain $i$ and the other relative to chains $j$. Figure I.4 shows that these two components vary locally, which is characteristic of a non-ideal system. However, taking into account the effects of compensation, chain $i$ does not undergo a particular force: each of its repeal units is surrounded indifferently by the repeat units of the same chain or different chains. This is the situation of ideal solutions, a situation analogous to the $\theta$ solvents: hence the chain is ideal because the excluded volume effects are negligible.

The mixed chains-segregated chains alternative has long been discussed. These two possibilities are schematically depicted in Figure 1.5.

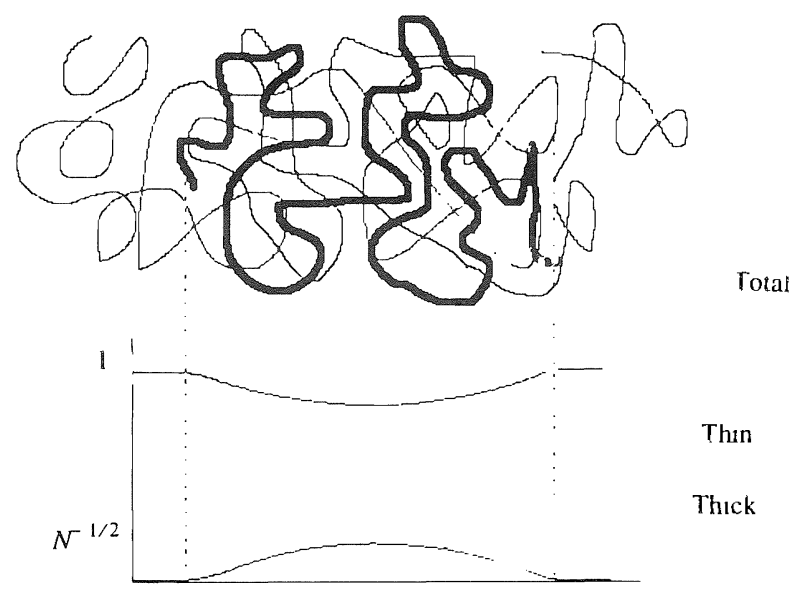

Fig. 1.4. Mixture of two macromolecules (thin and thick lines) and concentration of each along a spatial co-ordinate.

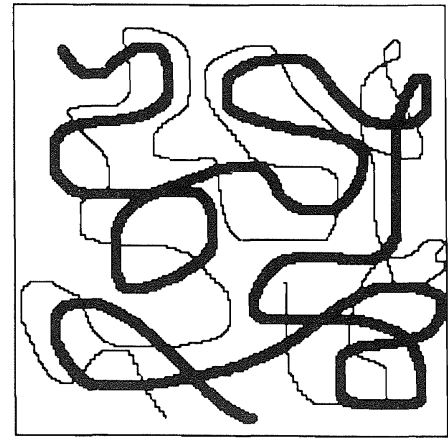

(a)

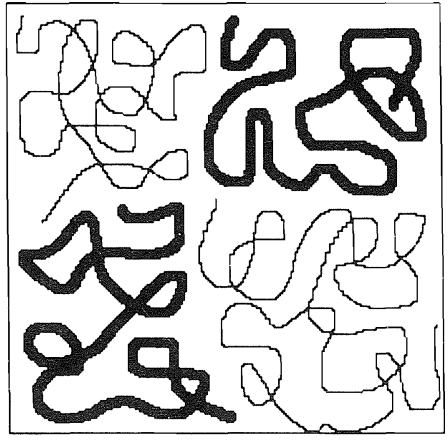

(b)

Fig. I.5. Mixture of macromolecules with interpenetration (a) or forming separated coils (b). 
In fact, both positions are physically possible depending on the dimensionality. Indeed the volume of the coil is of the order $N^{3 / 2} * \lambda^{3}$ whereas the volume of the matter forming a macromolecule is $N \lambda^{3}$, which is smaller by a factor of $N^{1 / 2}$; this signifies that in this volume of the coil a large number of macromolecules may be placed. On the other hand, in two dimensions, the volume of the coil would be of the order of $N \lambda^{3}$; in this case, it is equal to the volume of the matter constituting the macromolecule.

To summarise, the condensed matter in three dimensions, formed by a polymeric material must be seen as a succession of intermingled macromolecules. Each macromolecule exists like a coil having Flory's radius $R_{F} \approx N^{1 / 2}$ but in the same space a larger number of macromolecules (of the order of $N^{1 / 2}$ ) are found intermingled. Moreover, neutron scattering experiments carried out with macromolecules labelled with deuterium have confirmed that in a condensed state, macromolecular chains are ideal and Gaussian.

Naturally, such a succession in a supercooled liquid state is in metastable thermodynamic equilibrium. The thermal fluctuations thus permanently modify the conformations, the average value of $\lambda_{t}$ remaining well around its equilibrium value, which for a given polymer depends only on temperature. Moreover, Van der Waals bond form and break between the neighbouring structural units which, as we have seen, most probably pertain to different macromolecules.

\subsection{Intermolecular Interaction: Cohesion in Solid Polymers}

In the preceding section we described the interaction between neighbouring structural units. Though this interaction is weaker than covalent intermolecular interaction, the large number of these interactions along a macromolecular chain enhances their importance. Hence a decrease in temperature accompanies an increase in viscosity. Qualitatively, this increase in viscosity may be described as due to these two reasons:

- One, conformational: on the one hand, the average length (i.e., rigidity of the chain) increases and, on the other, the characteristic time of conformational changes $\tau_{t g}$ also increases;

- Two, related to intermolecular interactions: the number of bonds between structural units increases in proportion to the decrease in temperature.

Since the intermolecular interaction effects are not negligible, the characteristic time of conformational change, a process necessary for metastable equilibrium of the system, may not be represented by $\tau_{t g}$ but by time $\tau_{m o l}$, which must simultaneously take into account intra- and intermolecular barriers ( $\tau_{m o l}$ will be defined in Chapter II).

During a decrease in temperature the system maintains its equilibrium so long as the duration of observation (experimental time $t_{\text {exp }}$ ) is higher than $\tau_{m o l}$. Starting from the temperatrue at which $t_{\exp }$ is comparable and, for this case shorter than $\tau_{m o l}$, the configurational changes (conformational states and also arran- 
gement of each structural unit with respect to its neighbours) lack sufficient time to do so and the system loses its metastable equilibrium and becomes macroscopically out of equilibrium. At this temperature, denoted as glass transition temperature $T_{g}$, viscosity is of the order of $10^{11}-10^{12} \mathrm{~Pa} \cdot \mathrm{s}$; thus, at temperatures of the order of or smaller than $T_{g}$ the system is considered to be in a glassy solid state. This point will be discussed in detail in Chapter VII but here we shall explain the forces responsible for cohesion of this solid state.

The property essentially characterising this cohesion is cohesive energy density. It is generally defined by the parameter $\delta_{E}$ obtained according to the following expression:

$$
\delta_{E}=\left[N_{A} U_{m} / V\right]^{1 / 2},
$$

where $N_{A}$ is Avogadro's number; $U_{m}$ the mean value of the intermolecular interaction energy per structural unit: $V$ the molar volume.

The parameter $\delta_{E}$ is determined in various ways: measurement of heat of vaporisation, swelling in different solvents, measurement of viscosity of polymer solutions etc. Let us also mention the method of Small which consists of calculating $\delta_{E}$ from the molar attraction constants $F_{m a}$

$$
\delta_{E}=\frac{\sum F_{m a}}{V}
$$

Tables giving the values of $F_{m a}$ are available, from which we can ascertain $\delta_{E}$ for most polymers. From this it is possible to establish a relation between cohesive energy density and the properties of materials, such as the heat of polymer-solvent and polymer-polymer mixing. General textbooks on polymers discuss this subject. We deal here mainly with the elastic modulus of solid polymers.

The adiabatic compressive modulus is related to the potential energy of intermolecular interaction (molar value $U_{m o l}$ ) by the following expressions:

$$
B=V \frac{d^{2} U_{m o l}}{d V^{2}}
$$

In other words, the compressive modulus is proportional to the curvature of the potential of interaction. Considering the Lennard-Jones potential (see Sec. 1.1, relation (I.1)), we may estimate the value of $B$ for the case of amorphous polymers by using the following information:

- This potential corresponds to Van der Waals interactions between neighbouring structural units;

- Determination of the parameters $C_{r e p}$ and $C_{\text {atr }}$ is possible by taking into account the fact that at $d U_{m o} / d r=0$ the energy of interaction is $U_{m}$ and the distance between structural units is $\lambda$ (we would take the reasonable values of $-0.4 \mathrm{eV}$ and $5 \AA$ respectively); 
- The molar volume $V$ is taken as equal to $N_{A} \times \lambda^{3}$ and $U_{m o l}=1 / 2 N_{A} U_{m}$.

These expressions then allow us to calculate:

$$
B=-4 U_{m} / \lambda^{3} \text {. }
$$

giving, with the aforesaid values, $B \approx 2 \mathrm{GPa}$.

It is interesting to note that Grüneisen equation

$$
B \approx C_{v} / 3 \alpha_{l} V_{s}
$$

where $\alpha_{l}$ is the coefficient of linear thermal expansion; $C_{v}$ the specific heat; $V_{s}$ the volume mass, leading to $B \approx 3 \mathrm{GPa}$.

Finally, the relation between $B$, shear modulus $G$ and Poisson ratio $v_{p}$ (mechanics of continuous media):

$$
G=\frac{3}{2} \times \frac{1-2 v_{p}}{1+v_{p}} \times B
$$

leads us to a value of $G: G \approx 1 \mathrm{GPa}$.

All these values are quite comparable to experimental data, even when considering the instantaneous moduli (or at infinite frequency), experimentally obtained at low temperature or (and) at ultrasonic frequencies (greater than $\mathrm{MHz}$ ). This agreement emphasises the fact that the cohesion of a polymer solid results essentially from the effect of interactions of the Van der Waals type between neighbouring structural units; the intermolecular forces thus make a negligible contribution to the property 'elastic modulus' of solid amorphous polymers. This point of view is further reinforced if we consider the situation wherein covalent bonds are involved during the application of a stress. This is the case when the chains are highly oriented (example: high modulus polymeric fibres) or even form linear chain crystallites (example: polyethylene). The longitudinal modulus in the chain direction may then attain or even surpass $100 \mathrm{GPa}$ !

To summarise. solid amorphous polymers can be considered a disordered arrangement of structural units whose cohesion is provided by the Van der Waals forces exerted between the latter. In the next section we describe some aspects of the microstructure of this arrangement.

\subsection{Concept of Physical Defects in Solid Amorphous Polymers}

In the preceding section we showed how amorphous polymers exist in a solid state: each structural unit is part of a chain and concomitantly finds itself inserted in a matrix formed by other structural units. Nevertheless, it is clear that all these structural units do not occur in strictly identical situations. We may anticipate a distinction between the repeat units for several reasons.

- First of all, the chemical environment may be different. This is the case, for example, between a repeat unit at the chain end and that in the chain, or between a repeat unit forming a site for branching and that constituting a linear chain, or even with structural units with anomalies of succession (head to head, head to tail etc.). 
- Thereafter, the physical situation of each repeat unit or, more precisely, the interactions with the environment may not be identical. This situation leads to spatial fluctuations inherent to the disordered state of amorphous polymers. The interactions mentioned here are, on the one hand, intramolecular (which governs the macromolecular conformation) and on the other, intermolecular (hence it is necessary to examine the level of Van der Waals energy characteristic of this repeat unit in interaction with its neighbours other than those which precede or succeed it in the chains).

Based on these considerations, we introduce the concept of physical defects', similar to the concept widely used in physical metallurgy. For this purpose let us first recall the concepts of 'forces' which have enabled the development of various models of defects. We shall then describe the elements which characterise the concept of 'quasi-point defects' in amorphous polymers. Finally, the concept of 'dislocation' will be introduced and the effect of direction that gives rise to this concept in the case of disordered matier discussed.

\subsection{Concept of point defects}

(a) Various approaches: A large number of publications describe the disordered arrangement of a succession of structural units and unveil the idea of 'heterogeneity' at the molecular level. In the case of amorphous polymers. Gibbs and Di Marzio used a quasi-network model and calculated the partition function of a system of $n$ chains, each comprising $x$ monomers. They suggested that the free enthalpy of this system in thermodynamic equilibrium (supercooled liquid) depends:

- On the distribution of bond between the 'trans' and 'gauche' states:

- On the absence of monomers at a certain number of junction points of the quasi-network (i.e., the rupture of Van der Waals bonds between structural units normally in contact).

A knowledge of this partition function is mandatory for understanding all the properties of the system. Yet in spite of the simplifying assumptions employed by Gibbs and Di Marzio, their work shows that the relations are very complex. The conclusion to such an approach is that if the temperature is decreased. the supercooled liquid finds its configurational entropy becomes zero at a critical temperature $T_{2}$. where a second-order phase transition occurs. In this range of temperature the molecular mobility is so weak that the system de facto hecomes frozen to such an extent that this phase transition cannot be experimentally observed.

This approach distinguishes between structural units with the least free enthalpy (trans conformation, maximum Van der Waals bonds with neighbours) and those with very high free enthalpy. The latter describe structural defects which will be discussed later. However, this concept is restricted by the fact that the resultant phase transition remains hypothetical, as the structure of the phase obtained by transformation of the supercooled liauid is totally ignored. 
A very different conclusion is drawn from the works of Egami and Vitek. They carried out a numerical simulation of the atomic arrangement of metallic solids from the hypothesis of random compact arrangement of atoms bonded by pair potential $U\left(\left|r_{i j}\right|\right)$. The total energy of interaction is given by:

$$
U=1 / 2 \sum_{i, j \neq 1} U\left(\left|r_{i j}\right|\right)
$$

A uniform deformation $\varepsilon^{\alpha \beta}$ accompanies a variation of this energy of interaction given by:

$$
\Delta U=1 / 2 \sum_{\alpha=\beta=1}^{3} \sum_{i, j \neq i} \frac{d U\left(\left|r_{i j}\right|\right)}{d\left|r_{i j}\right|} \frac{r_{i j}^{\alpha} \times r_{i j}^{\beta}}{\left|r_{i j}\right|} \varepsilon^{\alpha \beta} .
$$

Introducing the tensor of 'atomic stresses' $\sigma_{i}^{\alpha \beta}$ corresponding to the volume $\Omega_{i}$ of atom $i$, we also have:

$$
\Delta U=\sum_{\alpha=\beta=1}^{3} \sum_{t} \Omega_{i} \sigma_{i}^{\alpha \beta} \varepsilon^{\alpha \beta}
$$

By equating, we find the tensor of atomic stresses:

$$
\sigma_{l}^{\alpha \beta}=\frac{1}{2 \Omega_{i}} \sum \frac{d U\left(\left|r_{i j}\right|\right)}{d\left|r_{i j}\right|} \frac{r_{i j}^{\alpha} \times r_{i j}^{\beta}}{\left|r_{i j}\right|} .
$$

This tensor can be resolved into its two invariants expressed in terms of the components in principal directions by:

- the trace $T_{r}=1 / 3\left(\sigma_{1}+\sigma_{2}+\sigma_{3}\right)$,

- the deviator $D_{e}=\left\{1 / 3\left[\left(\sigma_{1}-\sigma_{2}\right)^{2} / 2+\left(\sigma_{1}-\sigma_{3}\right)^{2} / 2+\left(\sigma_{2}-\sigma_{3}\right)^{2} / 2\right]\right\}^{1 / 2}$.

Numerical calculations extended to the models of more than a thousand atoms give rise to three types of heterogeneities or defects of the size of domains of the order of a dozen atoms, for which:

$-T_{r}$ is high and the value positive: $n$ defects

- $T_{r}$ is high but the value negative: $p$ defects

- $D_{e}$ is high: $\tau$ defects.

Naturally, the values of $T_{r}$ and $D_{e}$ are distributed but the 'defect' sites correspond to the tails of distribution. The $n$ and $p$ defects must be considered fluctuations of density, respectively negative and positive. Such defects are therefore equivalent to the vacancies and interstitial gaps in the crystalline solids but are distributed in their volume over and above the atomic volume. The $\tau$ defects are the zones of high shear. The first type of defects is involved in diffusion mechanisms whereas the second ones could intervene in the process of inelastic deformation.

In principle, there is no opposition to the argument that calculations should be done in the case of amorphous polymers conforming to a disordered arrangement of 
structural units for which a spherical shape is assumed and the hypothesis wherein only intermolecular interaction is taken into account with the potential $U\left(\left|r_{i j}\right|\right)$.

In the case of metallic liquids, however, Cunat developed another model considering that these liquids consist of a mixture of atoms, distinguishable by nature and number of degrees of freedom. Thus we have with an increase in level of enthalpy:

- 3 vibrational degrees of freedom,

-2 vibrational and 1 translational,

- I vibrational and 2 translational,

-3 translational degrees of freedom.

A 'physicochemical' description of the liquid is thus presented in terms of thermodynamic equilibrium for each family, which in turn is dependent on temperature. Consequently, when the temperature decreases, the equilibrium is changed and the population of each family of atoms as well as their mobility increases. The glass transition temperature thus appears as that wherein the various equilibrium processes coincide or. more precisely, the temperature for which the internal time scale of chemical equilibrium processes between the different types of atoms becomes greater than that of the duration of the experiment.

As in the case of the works of Egami and Vitek, the model of Cunat considers a transposition possible in the case of amorphous polymers, but in all other cases intermolecular effects are not taken into account. Furthermore, the kinetic properties of these 'defects' (atomic or molecular mobility) remain quite phenomenological.

(b) Quasi-point defects in amorphous polymers: The preceding remarks enable us to describe the hypotheses which appear most appropriate for the case of amorphous polymers.

First, we shall describe the representation of these materials in terms of disordered arrangement of structural units, each being in a cage formed by neighbours. One such cage can be described by a Voronoï polyhedron to be compared to a Wigner Seitz polyhedron (Fig. I.6a); the enthalpy $H$ of this structural unit can then be represented as a function of the dimension $\Phi$ of the cage by a curve which qualitatively has necessarily the shape shown in Figure 1.6b. Further development of this qualitative representation is difficult: the function $H(\Phi)$ comprises not only the intermolecular interaction which may be described by the Lennard-Jones potential, but also the intermolecular interaction with two neighbouring structural units of the same molecular chain. It is also difficult to give a function $H(\Phi)$ capable of describing these two types of interaction simultaneously; we have taken the curve shown in Figure I.6(b).

We term as quasi-point defects those sites formed from a structural unit and its first neighbours (the above-mentioned cage) whose free energy is higher than the average value of the succession of structural units. 


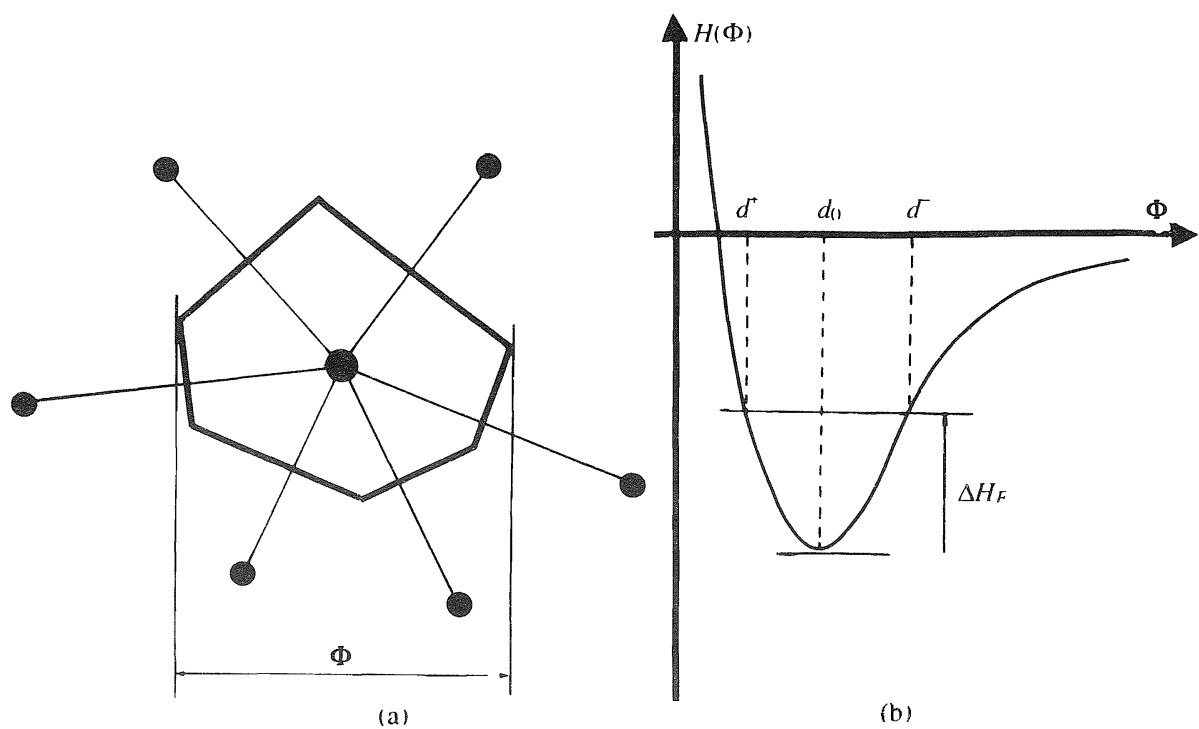

Fig. 1.6. Arrangement of a structural unit and its first neighbours and formation of a Voronoï polyhedron of dimension $\Phi(a)$ : variation of the enthalpy of interaction of the actual units of the polyhedron as a function of $\Phi(b)$.

To calculate the concentration of these defects, we proceed, on the one hand, by considering the system in thermodynamic equilibrium (supercooled liquid) and, on the other, distinguishing two types of structural units:

$-N_{A}-N_{d}$ the number of units having the least level of enthalpy (minimum of the $H(\Phi)$ curve or near it);

$-N_{d}$ the number of units forming with their neighbours a quasi-point defect and with which one must attribute the increase in free enthalpy:

$$
\Delta G_{F}=\Delta H_{F}-T \Delta S_{F},
$$

where $\Delta H_{F}$ is increment in enthalpy due to broken or perturbed Van der Waals bonds and to the formation of intramolecular bonds: $\Delta S_{F}$ the increment in entropy related to formation of the defect.

The number of defects $N_{d}$ (i.e., the concentration $C_{d}=N_{d} / N_{A}$ ) is obtained by minimising the function:

$$
\Delta G=N_{d} \Delta G_{F}-T \Delta S_{m},
$$

where $\Delta S_{m}=k \log \left[N_{A} ! /\left(N_{A}-N_{d}\right) ! N_{d} !\right]$ is the entropy of mixing of $N_{d}$ defects in $N_{A}$ possible positions. The calculations lead us to:

$$
C_{d}=\frac{1}{1+\exp \left(-\Delta S_{F} / k\right) \times \exp \left(\Delta H_{F} / k T\right)} .
$$


The advantage of such an approach lies in the fact that we may determine $\Delta H_{F}$ and $\Delta S_{F}$ from calorimetric quantities. Indeed, when we consider the classical scheme of Figure I.7(a) for one mole, i.e., $N_{A}$ number of structural units, we have a linear form

$$
\Delta H(T)=\Delta C_{p}\left(T-T_{1}\right),
$$

where $\Delta C_{p}$ is the difference between $C_{p}$ (supercooled liquid) and $C_{p}$ (glass) (values in molar quantities). From the preceding definitions we may write:

$$
\Delta H(T)=\Delta H_{F} N_{A} C_{d}
$$

This determination must be done in the temperature range close to $T_{g}$ for which the preceding three relations enable us to calculate the desired quantities $\Delta H_{F}$ and $\Delta S_{F}$. This goal can be achieved by several methods:

(a) Figure I.7(a) shows that the configurational enthalpy becomes zero at $T=T_{1}$. Noting that $T_{1} \approx 0.8$ to $0.9 T_{2}$. the temperature at which the entropy of configuration becomes zero, as suggested by Adam and Gibbs, we obtain directly from the relations ( $1.7 \mathrm{a} . \mathrm{b}$ and $\mathrm{c}$ ) the following expressions:

$$
\begin{gathered}
\Delta H_{F}=c_{T} k T_{g}+\frac{\Delta C_{p} \times T_{g}}{N_{A} \times c_{T}}: \\
\Delta S_{F}=\frac{\Delta H_{F}}{T_{g}}-k \ln \frac{1-C_{d}\left(T_{g}\right)}{C_{d}\left(T_{g}\right)} ; \\
C_{d}\left(T_{g}\right)=\frac{\Delta C_{p} \times T_{g}}{N_{A} c_{T} \Delta H_{F}} .
\end{gathered}
$$

These expressions contain the constant $c_{T}=T_{g}\left(T_{g}-T_{1}\right)$. which depends on $T_{g}$; considering the $T_{g}$ measured by standard technics, Adam and Gibbs showed

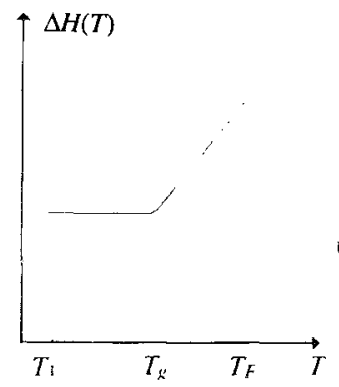

(a)

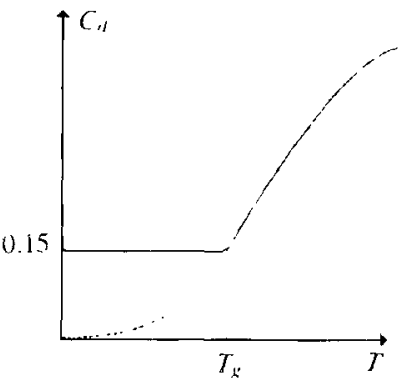

(b)

Fig. 1.7. Schematic variation of enthalpy of a glassy system with temperature (a) and representation of the curve $C_{d}(T)$ in thermodynamic equilibrium (solid curve) or after extrapolation at $T_{R}$ (broken line curve) (b). 
for an entire series of amorphous polymers a value of $T_{2} / T_{g} \sim 0.6$ to 0.7 , leading to $c_{T}$ of the order of 2 to 3 .

(b) Another method consists of starting from the expression of configurational entropy:

$$
\begin{aligned}
S(T)= & N_{A} \Delta S_{F} C_{d}-N_{A} k C_{d} \ln C_{d}- \\
& N_{A} k\left(1-C_{d}\right) \ln \left(1-C_{d}\right),
\end{aligned}
$$

which can be represented with good precision by the following relation:

$$
S(T)=a_{s} \times C_{d}(T)^{m_{s}} .
$$

If we recall that Adam and Gibbs used the expression $S(T)=\Delta C_{p} \ln \left(T / T_{2}\right)$. we may through equating draw three relations giving $S(T)$ :

$$
C_{d}\left(T_{g}\right)=\left[\frac{S\left(T_{g}\right)}{a_{s}}\right]^{1 / m_{s}}=\left[\frac{\Delta C_{p} \ln \left(T_{g} / T_{2}\right)}{a_{s}}\right]^{1 / m_{s}}
$$

With relations (1.7), we obtain:

$$
\begin{gathered}
\Delta H_{F}=k T_{g} \times\left[\frac{\Delta C_{p} / R}{C_{d}\left(T_{g}\right) \times\left(1-C_{d}\left(T_{g}\right)\right)}\right] \\
\Delta S_{F}=\frac{\Delta H_{F}}{T_{g}}-k \ln \left[1 / C_{d}\left(T_{g}\right)-1\right] .
\end{gathered}
$$

(c) The third method is based on the following argument: as we approach $T_{g}$ we observe a value of $\Delta C_{p}$ which does not vary with temperature. This leads us to the condition wherein $T_{g}$ is situated towards the point of inflection of the curve $C_{d}(T)$ (see Fig. I.7(b)). To equations (1.7) the condition $d^{2} C_{d}(T) / d T^{2}=0$ is added and all these equations give:

$$
\begin{gathered}
C_{d}\left(T_{g}\right)=0.5 \times\left[1-\left(1+\Delta C_{p} / R\right)^{-0.5}\right] ; \\
\Delta H_{F}=k T_{g} \times 2 /\left(1-2 C_{d}\left(T_{g}\right)\right) ; \\
\Delta S_{F}=\frac{2 k}{1-2 C_{d}\left(T_{g}\right)}-k \ln \left[1 / C_{d}\left(T_{g}\right)-1\right] .
\end{gathered}
$$

Remark: from $\Delta C_{p}=d \Delta H(T) / d T$, we obtain by using equations (I.7a) and $(1.7 c)$ :

$$
\frac{\Delta C_{p}}{N_{A}}=\frac{\Delta H_{F}^{2}}{k T_{g}^{2}} \times C_{d}\left(T_{g}\right) \times\left[1-C_{d}\left(T_{g}\right)\right] .
$$

This equation has only a single solution for $\Delta H_{F}$ for any given value of $\Delta S_{F}$; the pair of values of $\Delta H_{F}$ and $\Delta S_{F}$ thus obtained correspond only to those resulting from the condition $d^{2} C_{d}(T) / d T^{2}=0$. 
Thus it is useful to compare the results obtained from equations $(1.8,1.9$ and 1.10). For this purpose let us consider the case of a glassy state of glycerol; the experiment gives $\Delta C_{p}=19.5 \mathrm{cal} /($ mole $\cdot K)$ or, with 6 degrees of freedom per molecule (see later), $13.6 \mathrm{~J} /\left(\right.$ mole $\cdot d^{\circ}$ liberty $\left.\mathrm{K}\right) ; T_{g} \approx 185 \mathrm{~K}$ and $T_{2} \approx 127 \mathrm{~K}$. These experimental values lead to $0.139-0.05 \mathrm{eV}$ and 1.876 (method a), $0.131-0.06 \mathrm{eV}$ and 1.888 (method $b$ ), 0.192-0.052 and 1.808 (method $c$ ) for respectively $C_{d}\left(T_{g}\right.$ ), $\Delta H_{F}$ and $\Delta S_{F} / k$. It has been verified that the values adopted remain comparable irrespective of the method used.

It must be noted that the defects thus arising are the sites at which fluctuations of density occur. Moreover, these fluctuations can be either positive or negative: in this way they are of the same nature as the $p$ and $n$ defects described above. But in all cases the defect corresponds to a positive fluctuation of enthalpy (and of entropy). It must be emphasised that a defect of negative fluctuation of density does not differ from a domain of matter of large free volume. The concept of defect introduced here thus includes not only the idea of free volume, but also that of 'anti-free volume' sometimes encountered in the case of metallic glasses. Referring to the case of crystals, we may say that the quasi-point defects in the amorphous matter are equivalent to the distributed 'vacancies' and 'interstitials'. With respect to the notion of free volume, the concept of quasi-point defect has the great advantage of taking into account the effects of intermolecular interaction, which are significant in the temperature range near $T_{g}$.

Consider, as an example. the case of glassy selenium, a particular solid polymer (repeat unit reduces to an atom) for which the values of various properties are well established. With $\Delta C_{p}=15.9 \mathrm{~J} /(\mathrm{mole} \cdot \mathrm{K})$ and $T_{g}=300 \mathrm{~K}$, we obtain from equations (I.8) $\Delta S_{F}=2.3 k$ and $\Delta H_{F}=0.114 \mathrm{eV}$. Figure I.7(b) shows the variation of $C_{d}$ with temperature by assuming that the system remains in metastable thermodynamic equilibrium (supercooled liquid). It may be noted that $C_{d}\left(T_{g}\right)$ is close to 0.15 , a value typical of the numerous materials studied, as we shall see in subsequent chapters.

To judge the validity of such an approach in the case of selenium. it is possible to draw from the results thus obtained various values amenable to comparison with experimental values. Let us see first of all the enthalpy of fusion: Figure I.7 shows us that it is of the order of $N_{A} \times C_{d}\left(T_{F}\right) \times \Delta H_{F}$, being 4.6 $\mathrm{kJ} /$ mole; experimentally we obtain $6.2 \mathrm{~kJ} /$ mole. Now let us consider the difference in density between the glassy and crystalline states: admitting that the perturbed arrangement of repeat units shows a loss of compactness by $16 \%$, as predicted by the Bernal model of random packing of hard spheres, the difference in relative specific volume $\Delta V / V$ between glass and 'crystal' is then

$$
\Delta V / V=C_{d}\left(T_{g}\right) \times 0.16=0.018
$$


Experimentally we observe 0.024 for monoclinic crystal. Finally, we may calculate $\Delta \alpha_{\nu}$, the difference in coefficient of thermal expansion between supercooled liquid and glass:

$$
\Delta \alpha_{\nu}=\frac{1}{V_{l q q}} \frac{d V_{\text {liq }}}{d T}-\frac{1}{V_{\text {glass }}} \frac{d V_{\text {glass }}}{d T} \frac{1}{V} \frac{d \Delta V}{d T}
$$

or

$$
\Delta \alpha_{v}=0.16 \frac{d C_{d}}{d T}
$$

The equation giving $C_{d}$ thus gives $\Delta \alpha_{v} \approx 2.1 \times 10^{-4}$. whereas experimentally we have $2.7 \times 10^{-4}$.

The difference between these estimations and the experimental values is less than $30 \%$ in all cases. We may thus admit that such a description can reasonably take into account the various properties of the system (in the present case. selenium). But it is clear that this approach is too simplified and it is possible to improve several aspects of this presentation.

For example, there is no reason to take a single value of $\Delta H_{F}$ (as of $\Delta S_{F}$ ) in a disordered manner. The calculations of Vitek and Egami described above suggesi rather a distribution of values. Thus by admitting $i$ discrete values for $\Delta H_{F}$ and $\Delta S_{F}$, we arrive at $i$ families of defects, each family having the concentration $C_{l}$ given by:

$$
C_{\imath}=\frac{\exp \left[\Delta S_{F}(i) / k\right] \times \exp \left[-\Delta H_{F}(i) / k T\right]}{1+\sum_{i} \exp \left[\Delta S_{F}(i) / k\right] \times \exp \left[-\Delta H_{F}(i) / k t\right]},
$$

where $C_{d}=\sum C_{i}$

It is then possible to numerically calculate $C_{d}$ by taking $1<i<q$ and the simplest relations between $\Delta H_{F}(i)$ or $\Delta S_{F}(i)$ and $i$. We may, for example, consider the linear relations:

$$
\begin{aligned}
\Delta H_{F}(i) & =\Delta H_{0}+i \times H_{1} ; \\
\Delta S_{F}(i) & =\Delta S_{0}+i \times S_{1} .
\end{aligned}
$$

All the parameters may be adjusted with reference to $\Delta C_{p}$ and $T_{g}$ with eventually $T_{2}$, as explained above.

We may generalise the approach developed above by applying the laws of statistical physics to a population of degrees of freedom. This leads us to consider that the notion of defect is applicable not to the assembly of repeat units, but rather to each of the degrees of freedom of the type of rotation around the axis formed by the covalent bonds. Given these conditions, it is necessary to attempt to ex lude the number $n_{d l}$ from these degrees of freedom (liberty) per repeat unit 
and attribute to $\Delta C_{p} / n_{d l}$ the contribution of each degree of freedom to the excess specific heat. In this regard let us mention the work of Muser who observed that for the most classical amorphous polymers, $\Delta C_{p} / n_{d l}$ is quite close to $(3 / 2) R$, the value conforming to that given by thermodynamics. We also know that the value $\Delta S_{F} \approx 1.8$ to $2 k$ could show the number of neighbours (given by $\exp \left(\Delta S_{F} / k\right) \approx 6$ to 8 ) susceptible to implication in the intermolecular bonds perturbed during the formation of the quasi-point defect. Of the remaining, $\Delta H_{F}$. which is generally of the order of $3 k T_{g}$, has a value which could correspond to the energy of a Van der Waals bond (this point will be rediscussed in Chapter VII).

So, now. let us emphasise that the approach presented above is based on the concept according to which the system is formed of a compact disordered packing of completely identical structural units. Such an idea. acceptable in the case of molecular. metallic glasses. particularly the oxide glasses. becomes disputable for polymer systems. Indeed, besides the fact that the repeat units may be complex, we must consider that three types of these units exist in the macromolecular arrangement:

- the structural units in the middle of the linear chain,

- those at the ends of the chain,

- the structural units in the middle of the chain on which branching occurs (branching sites).

Under these conditions it is reasonable to use only the expressions shown above for completely linear chains of high molecular weight. In the contrary case, it is possible to modify these relations in the following manner: for an arrangement of $N_{A}$ structural units forming chains, each of which has $X$ units and by assuming that there is a number of branching sites corresponding to the fraction $y$ of the total number of structural units, the concentration of quasi-point defects is given by:

$$
\begin{aligned}
C_{d} & =\frac{2 / X}{1+\exp \left(-\Delta s_{f} / k\right) \times \exp \left(\Delta h_{f} / k T\right)} \\
& +\frac{1-2 / X-y}{1+\exp \left(-\Delta S_{F} / k\right) \times \exp \left(\Delta H_{F} / k T\right)},
\end{aligned}
$$

where $\Delta s_{f}$ and $\Delta h_{f}$ are entropy and enthalpy of creation of defects of structural units at the chain ends. It must be noted that these parameters are, in principle, experimentally assessable from the entropy and enthalpy of the condensed phase which would be formed from structural units of the same chemical nature before polymerisation (monomers). This new relation becomes quite equivalent to the preceding one when $X$ is large and $y$ is small.

In conclusion, we must emphasise that interest in the concept of quasi-point defects, just now introduced, is related to the clarifications that it enables in the analysis of various behaviours of solid polymers-in the first place molecular mobility, as will be shown in Chapter II, but also and mainly, when an analysis of 
phenomena of inelastic deformation observed in amorphous polymers (Chapters IV and V) is desired.

\subsubsection{Linear defects}

No one doubts the existence of dislocations in crystals: transmission electron microscopy and $\mathrm{X}$-ray topography (Lang method) provide highly varied observations. High resolution electron microscopy has even made possible determination of the structure at the heart of the dislocations. This significant advance in the area of physics of crystal defects has had an unexpected consequence: the belief, hotly disputed, that the concept of 'dislocation' is valid only in the case of crystalline solids. Is such a conviction justified? We shall attempt to answer two questions here:

- Can the concept of dislocation be applied to the case of amorphous material?

- If yes, how is it to be defined and what would be its use?

Let us first of all recall the conditions under which the concept was introduced. The first works were, in fact in the area of mechanics of continuous media (Volterra, 1907; Love, 1922). The objective of these studies was to determine the state of stress established after several cuttings done in matter considered to be a continuous, elastic and isotropic medium, then to reconstitute the continuity of this matter by displacement or/and rotation of the cut surfaces. Figure I.8 represents the various possible cases, which may be separated into two groups:

- Reconstitution of the continuity of the matter by translation of the cut surfaces (b, $c$ and $d$ in Fig. I.8). This axis of the cylinder of reconstituted matter is the line called 'dislocation'.

- Reconstitution by rotation of the cut surfaces (e, $f$ and $g$ in Fig. I.8) wherein linear defects designated 'disclination' are obtained.

This concept of dislocation was applied much later to crystal: edge dislocation (Polyani, Taylor, Orowan from 1923 to 1934) and screw dislocation (Orowan and Burgers from 1923 to 1939) were geometrically defined thanks to identification of the displacement vector of the cut surfaces (denoted in subsequent text by $\vec{v}_{d}$ ) as the translation vector of a crystal lattice (perfect dislocation); such a vector, called Burgers vector, is thus like an identity card of the 'dislocation' defect.

In the hypothesis of continuous medium, the dislocation appears to be a line of singularity of stress. In a real crystal the situation differs; the bonds are perturbed along the linear defect and we can then distinguish:

- The matter at medium and long distance from the line of defect. The perturbation of the bonds is moderated and expressed by the existence of a deformation and thus by a stress field whose nature depends on the type of dislocation (see in this regard specialised works, for example Dislocations by J. Friedel); 

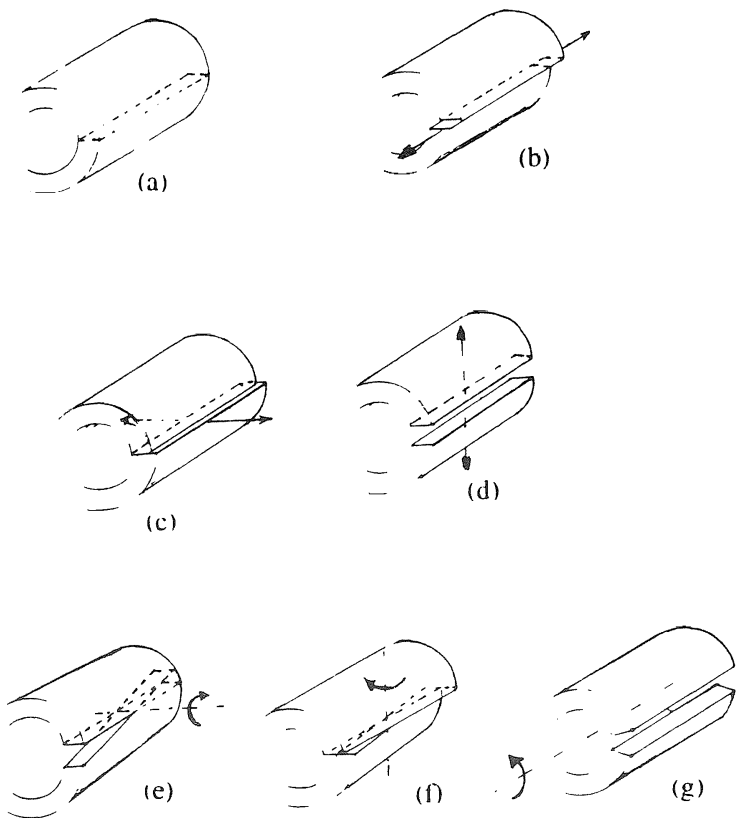

Fig. 1.8. Tube of matter with plane of cutting (a); translation with cut surfaces: dislocations (b to d): rotation of cul surfaces: disclinations (e to g).

- At a short distance. on the contrary, the perturbation of the bonds may be such that the order of atomic arrangement disappears; this is the heart of the dislocation.

Analysis of the structure of the heart of dislocations remains a difficult problem in spite of the advent of high-resolution electron microscopy. Let us consider here the representation given in Figure 1.9. Introduction of the concept of infinitesimal dislocation of the shear vector equal to $d \vec{v}_{d}$ allows us to write in accord with Figure I.9(a):

$$
\int_{0}^{\infty}\left(\frac{\vec{d} v_{d}}{d x}\right) d x=\vec{v}_{d} .
$$

Figure I.9(b) suggests these two possibilities:

- When $d v d d x$ is zero at all points except at $x=0$ where this quantity acquires an infinite value, we have a dislocation of Volterra (case of continuous media; existence of singularity of stress);

- When $d v_{d} d d x$ varies continuously from 0 to pass a maximum value at $x=0$ before again tending towards zero, this is called the Peierls dislocation, corresponding to the situation observed in real crystals; $w$ represents in this case the width of the heart of the dislocation.

Now let us see how the concept of dislocation can be applied to glassy solids. 


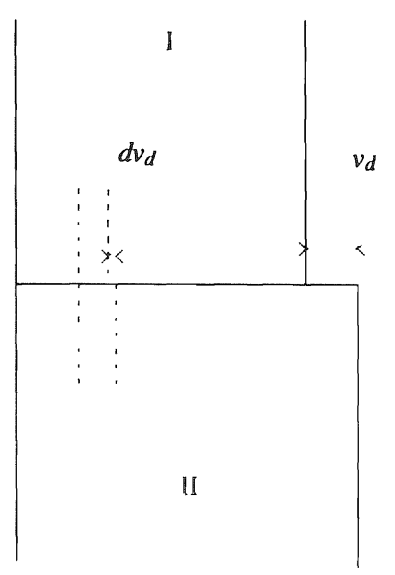

(a)

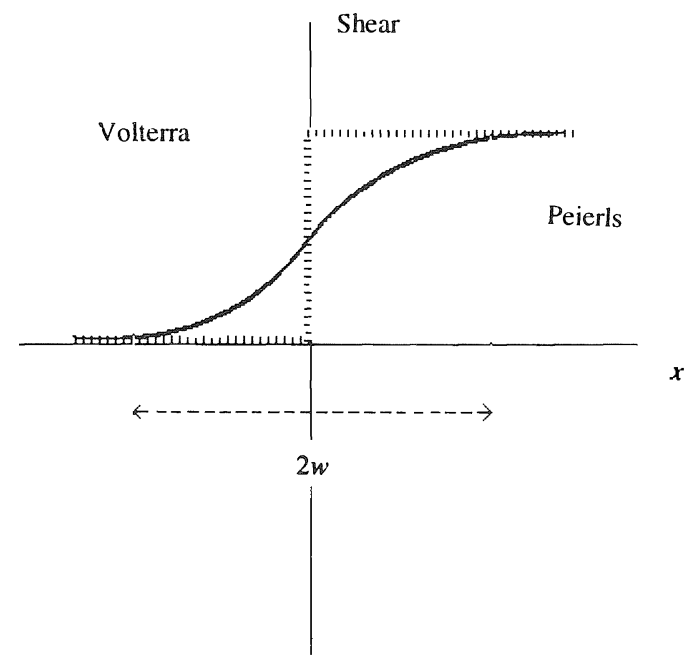

(b)

Fig. 1.9. (a) Shearing of part J of the solid with respect to part II by vector $v_{d}\left(d v_{d}\right.$ is the elementary shear between the sites of shear zero and $v_{d}$ ): (b) variation of shear from zero to $v_{d}$ from the line of dislocation.

Figure 1.9(b) shows that the sector of shearing is not constant in the heart of the dislocation. It is therefore natural to expect the same argument in the case of non-crystalline solids. Instead, as suggested by Gilman 15 years ago, the perturbation of bonds resulting from the dislocation defect cannot be the same all along this defect since these same bonds were already perturbed before the dislocation manifested. Figure I.10 illustrates this point in the case of the solid $\mathrm{SiO}_{2}$. We must therefore visualise for non-crystalline material non-conservation of the vector of shear along the linear defects. Furthermore, Figure I.11 shows the distinction between the dislocation of Volterra and the dislocation which might exist in the disordered matter or the Somigliana dislocation.

One way of defining the Somigliana disorder is to visualise that in the amorphous matter the perturbation of bonds related to the disorder may be represented by the microloops of dislocation whose shear vector $\Delta v_{d}$ varies randomly in space ( $\tau$ defects, as described by Vitek and Egami; see the preceding section). Shearing of one part of the solid with respect to another by the vector $v_{\text {shear }}$, leads to the reconstitution of the bonds, such as the disorder, which. though completely differeni, remains of the same nature on average, i.e., representable by the microloops of the randomisation vector $\Delta v^{\prime}{ }_{d}$. This corresponds to the situation in Figure I.11 wherein the loops of the Somigliana dislocation surround the domains for which the local shear is given by $v_{\text {shear }}+\Delta v^{\prime}{ }_{d}-\Delta v_{d}$.

In a similar manner, Figure I.12 enables a comparison of the Volterra dislocation (defects of zero width), the Peierls dislocation (defects of width $w$; the 


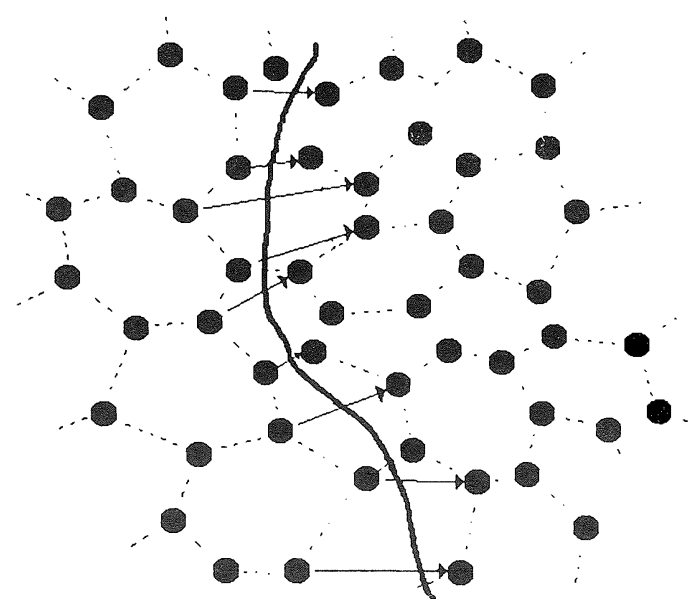

Fig. 1.10. Fluctuations of the vector of shear along the line of dislocation in the case of a disordered arrangement.

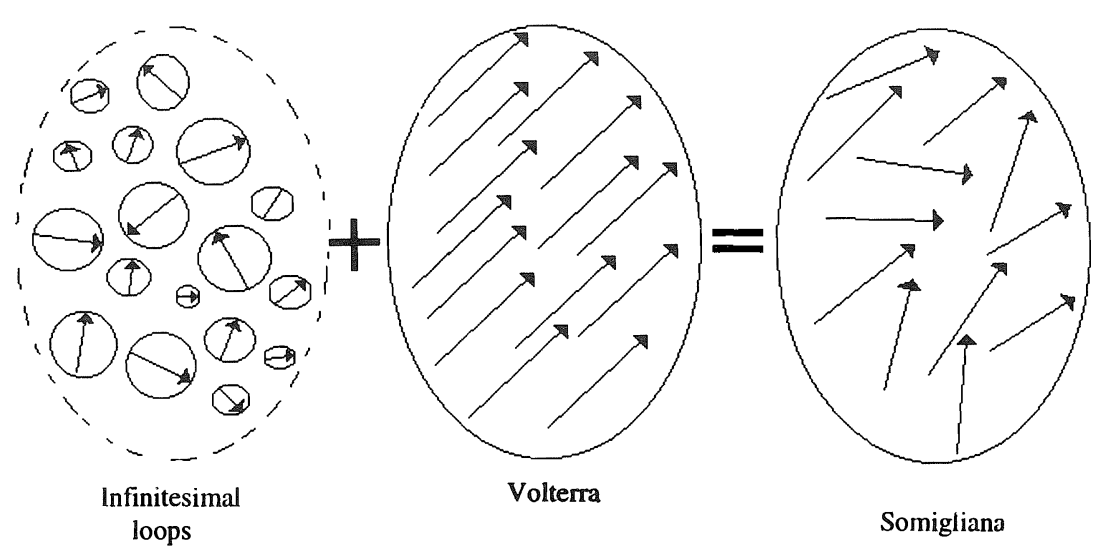

Fig. I.11. Dislocation of Somigliana is equivalent to the sum of a Voiterra dislocation and the total infinitesimal dislocation loops representing the disorder.

dislocation of vector $v_{d}$ corresponds on this width to the sum of infinitesimal dislocations) and the Somigliana dislocation (the dislocations also have a non-zero width but nonetheless add to the microloops associated with the disorder). In the schematic diagram of Figure I.12(b) we have shown the type of structural modification resulting from the displacement of the Somigliana dislocation.

Use of the concept of dislocation in non-crystalline solids evidently does not enable an analogy between this state of such matter and crystal. Through simple extrapolation, however, this concept becomes the simplest for describing the 


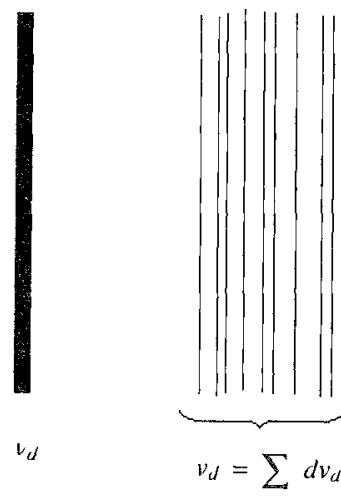

(a)

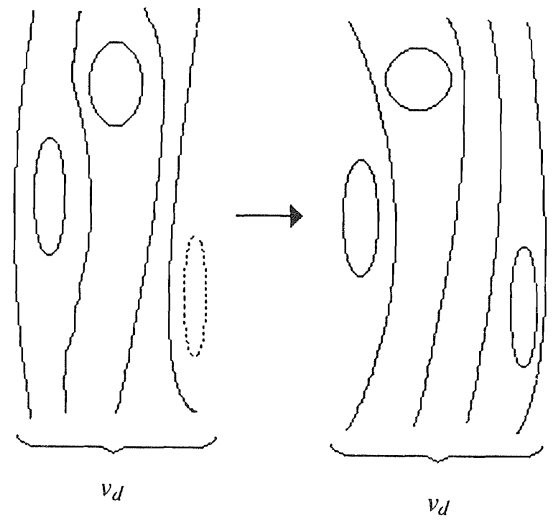

(b)

Fig. 1.12. (a) Equivalence between dislocation and the shear vector $v_{t}$ and the sum of dislocation of the vector $d v_{d}$ : (b) during motion of the linear defects in a disordered manner, it is necessary to visualise that each infinitesimal dislocation combines with the loops related to the disorder.

correlation between the movement of atoms (structural units) under the effect of the stimulus stress during the non-elastic deformation of a glassy solid. When the first local shearing is produced in a site of the glassy solid, the probability for a second local shearing to occur near the preceding one is much higher than that of the same event anywhere else, firstly because of the concentration of stress at the edge of the first localised shearing and, secondly, due to the fact that the constitution of local configurations is favourable to the extension of the shear. These two reasons hold true for both crystalline and amorphous matter. Hence this concept will be widely used in Chapters IV and V of this book.

Naturally, it must not be forgotten that the quasi-point defects described in the preceding section, for which hydrostatic stresses intervene, are added to the shear-stress-induced defects.

Coming back to Figure I.8, without going into details let us specify what corresponds to these disclinations. Figure I.13 shows the distinction between edge and twist disclinations corresponding to a rotation around an axis $\vec{\omega}$ parallel and perpendicular to the line of defect. The existence of such defects was suggested in the case of metallic glasses. Figure I.14 shows, for example, the arrangement of atoms corresponding to a dipole of edge disclination. This type of defect gives rise to that taken into consideration by Argon for developing a theory of plasticity of amorphous polymers based on the hypothesis of thermally activated nucleation of pairs called 'kink pairs'.

It is known that a combination of displacement and rotation may exist. Figure 1.15 shows two examples with the displacement vector $\vec{v}_{d}$ and rotation vector $\vec{\omega}$ perpendicular or parallel to the axis $\vec{d}$ of the defect. Such defects, termed dis- 


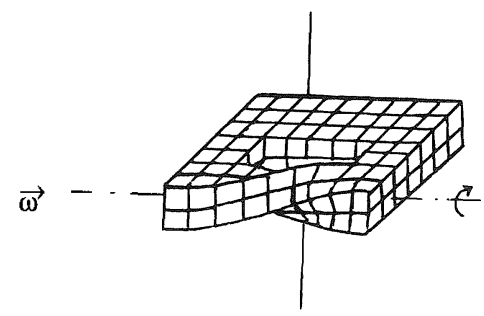

(a)

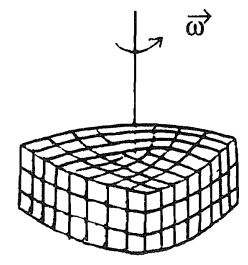

(b)

Fig. 1.13. Twist (a) and edge (b) disclinations.

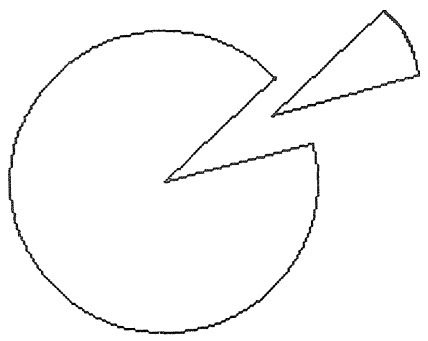

(a)

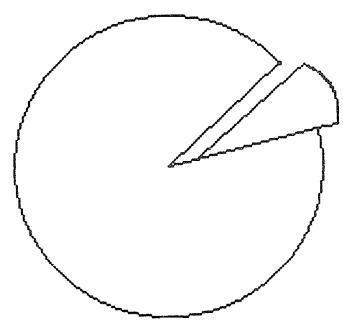

(b)

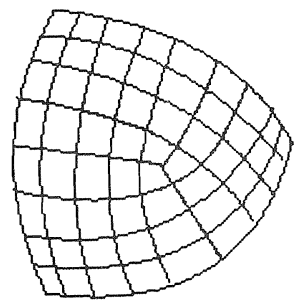

(c)

Fig. 1.14. (a) and (b) Formation of a dipole of disclinations by removal and emplacement of the matter but with a difference in position; (c) corresponding arrangement of atoms.

pirations, have been introduced to describe certain differences between the perfect crystalline arrangements of helical chains of polymers in crystallites. It must be noted that the interaction between dislocation and disclination appears under various aspects. Figure I. 16 shows the emission of dislocation by a dipole of edge disclination.

\section{CONCLUSION}

We have described in this chapter those aspects necessary for comprehension of the topics to be discussed in the chapters that follow. Besides the presentation of physicochemical properties of macromolecules, now well known in the case of polymers in solution or in the melt state', we have found the concept of defect applicable to solid amorphous polymers. In fact, such a concept must appear natural even in the case of non-crystalline solids about which these two fundamental concepts have been made quite clear.

(a) The concept of random perturbation of bonds associated with the notion of microfluctuation of density. The generalised disorder that occurs in non-crystal- 


\section{Structural Aspect of Polymers}

Dislocations. J. Friedel (ed.). Pergamon Press, Oxford, 1964.

Macromolecules-An Introduction to Polymer Science. F.A. Bovey and F.H. Winslow (eds.). Academic Press, Orlando, FLA, 1979.

Scaling Concepts in Polymer Physics. P.G. De Gennes (ed.). Cornell Univ. Press, Cornell, 1979.

The Element of Polymer Science and Engineering. A. Rudin (ed.). Academic Press, New York, 1982.

Adam, G. and J.M. Gibbs . J. Chem. Phys. 43 (1965): 139.

Argon, A.S. Phil. Mag. 28 (1973): 839.

Gibbs, G. and E. A. Di Marzio . J. Chem. Phys. 28 (1958): 373.

Gilman, J.J. J. Appl. Phys. 44 (1973).

Li, J.C.M. In: Metallic Glass, p. 224. Amer. Soc. Met. Metals Park. Ohio (1975).

Srolovitz, D. , K. Maeda . V. Vitek and T. Egami . Phil. Mag. 44 (1981): 847.

Cunat, C. Nancy (1985).

Muser, M. Diplôme Lyon (1990).

Trény, C. Grenoble (1984).

\section{Molecular Mobility in Amorphous Solid Polymers}

The Physics of Glassy Polymers. R.N. Haward (ed.). Mat. Science Series, App. Sci. Pub. Ltd., Barking, 1973.

Viscoelastic Properties of Polymers. J.D. Ferry (ed.). John Wiley \& Sons Inc., New York, 1983. Relaxations in Complex Systems. K.L. Ngai and G.B. Wright (eds.). Of. Nav. Research, Arlington, 1984.

Dynamics of Disordered Materials. D. Richter , A.J. Dianoux. W. Petry and J. Teixeira (eds.). Springer Verlag, Berlin, 1989.

Basic Feature of the Glassy State. J. Colmenero and A. Alegria (eds.). World Sci., Singapore, 1990.

Adam, G. and J.M. Gibbs . J. Chem. Phys. 43 (1965): 139.

Bernu, B. , J.P. Hansen , Y. Hiwatari and G. Pastore . Phys. Rev. A-36 (1987): 4891; Phys.

Rev. A-38 (1988): 454.

Cohen, M.H. and G.S. Grest . Phys. Rev. B, 20 (1979): 1077.

Cohen, M.H. and D. Turnbull . J. Chem. Phys. 31 (1959): 1164; J. Chem. Phys. 34 (1961): 120.

Glarum, S. J. Chem. Phys. 33 (1960): 639.

Goldstein, M. J. Chem. Phys. 51 (1969): 3728.

Johari, G. In: Plastic Deformation of Amorphous and Semi-crystalline Materials, p. 109. B.

Escaig and C. G'sell (eds.). Ed. de Phys., Les Ullis (1982).

Monnerie, L. In: Initiation à la chimie et a la physico-chimie macromoleculaires, vol. 8, p. 183.

Ed. GFP (1990).

Ngai, K.L. and C. White . Phys. Rev. B, 20 (1979): 2475.

Palmer, R.G. , D.L. Stein , E. Abrahams and P.W. Anderson . Phys. Rev. Lett. 53 (1984): 958.

Perez, J. J. de Phys. C, 10-12 (1985): 427.

Perez, J. , J. Y. Cavaillé , S. Etienne and C. Jourdan . Rev. Phys. Appl. 23 (1988): 125.

Shlesinger, M.F. Ann. Rev. Phys. Chem. 39 (1988): 269.

Roux, J.N. Lyon (1990). 


\section{Non-elastic Deformation of Solid Amorphous Polymers}

Mechanical Properties of Solid Polymers. I.M. Ward (ed.). J. Wiley \& Sons, New York, 1983. Plastic Deformation of Amorphous and Semi-crystalline Materials. B. Escaig and C. G'sell (eds.). Les Editions de Phyls, Les Ullis, 1982.

The Physics of Glassy Polymers. R.N. Haward (ed.). Appl. Sci. Pub. Ltd., London, 1973. Creep, Viscoelasticity and Creep Fracture in Solids, J. Gittus (ed.) Appl. Sci. Publ. Ltd., London, 1975.

Physical Aging in Amorphous Polymers and Other Materials. L.C.E. Struik (ed.). Elsevier, Amsterdam, 1978.

Argon, A.S. Phil. Mag. 28 (1973): 839.

Bauwens-Crowet, C. , J.C. Bauwens and G. Holmes . J. Polymer Sci. A2, 7 (1969): 735.

Bowden, P.B. and S. Raha . Phil. Mag. 29 (1974): 149.

Escaig, B. Ann. Phys. 4 (1978): 207.

Eyring, H. J. Chem. Phys. 4 (1936): 283.

G'Sell, C. and J.J. Jonas . J. Mat. Sci. 16 (1981): 1956.

Guiu, F. and P.L. Pratt . Phys. St. Sol. 6 (1964): 111.

Oleinik, E.F. , Salamatina, O.B. , Rudnev, S.N. and Shenogin, S.V. Polym. Sci. (Russia) 11 (1993): 1819.

Plazek, D.J. In: Relaxations in Complex Systems, p. 83, K.L. Ngai and G.B. Wright (eds.). Off. Nav. Res., Arlington (1984).

Robertson, R.E. J. Chem. Phys. 44 (1966): 3950.

Cavaillé, J.Y. Lyon (1987).

Etienne, S. Lyon (1985).

Gopez, A.J. Nancy (1983).

Lacabanne, C. Toulouse (1972).

Lefebvre, J.M. Lille (1983).

\section{Theoretical Approach of Non-elastic Deformation of Solid Amorphous Polymers}

The Physics of Glassy Polymers. R.N. Haward (ed.). Appl. Sci. Pub.. London, 1973.

Dislocations et Déformation plastique. P. Groh , L.P. Kubi $n$ and J.L. Martin (eds.). Ed. de Phys., Les Ullis, 1979.

Plastic Deformation of Amorphous and Semi-crystalline Materials. B. Escaig and C. G'sell (eds.). Ed. de Phys., Les Ullis, 1982.

Mechanical Properties of Solid Polymers. I.M. Ward . John Wiley, Chichester, 1983.

Argon, A.S. Phil. Mag. 28 (1973): 839.

Argon, A.S. and H.Y. Kuo . Mat. Sci. Engng. 39 (1979): 110.

Bowden, P.B. and S. Raha . Phil. Mag. 22(1974): 149.

Cavaillé, J.Y., J. Perez and C.P. Johari Phys. Rev.B. 39 (1989): 2411.

Maeda, K. and S. Takeuchi . Phil. Mag. A44 (1981): 643.

Perez, J. Acta Met. 32 (1984): 2163.

Perez, J. Rev. Phys. Appl. 21 (1986): 93.

Perez, J. , J.Y. Cavaillé , S. Etienne and C. Jourdan , Rev. Phys. Appl. 23 (1988): 125.

Spaepen, F. J. Non Cryst. Sol. 31 (1978).

Cavaillé, J.Y. Lyon (1987).

Etienne, S. Lyon (1985). 


\section{Mechanical Experiments: Interpretation of Results}

Bultel, C. , J.M. Lefebvre and B. Escaig . Polymer 24 (1983): 476.

Cavaillé, J.Y. and J. Perez . Macromol. Chem.. Macromol. Symp. 35/36 (1990): 405.

Cavaillé, J.Y. , J. Perez and G.P. Johari . Phys. Rev. B. 39 (1989): 2411.

G'sell, C. In: Strength of Metals and Alloys, p. 1943. H.J. McQueen, J.P. Bailon, J.I. Dickson , J.J. Jonas and M.G. Akben (eds). Permagon Press, Oxford (1986).

G'sell, C. , H. El Bari , J.Y. Cavaillé and G.P. Johari . Mat. ScL \& Eng., A110 (1989): 223.

Lefebvre, J.M. and B. Escaig . J. Mat. Sci. 20 (1985): 438.

Perez, J. Acta Met. 32 (1984): 2163.

Perez, J. Rev. Phys. Appl. 21 (1986): 93.

Perez, J. and J.Y. Cavaillé . J. Phys. Ill, 5 (1995): 791.

Perez, J. , J.Y. Cavaillé , S. Etienne and C. Jourdan . Rev. Phys. Appl. 23 (1988): 125.

EJ Bari, H. Nancy (1987).

Flores-Flores, R. INSA. Lyon (1994).

Ladouce, L. INSA. Lyon (1995).

Martinez Vega, J.J. Poitiers (1986).

Muraciole, J.M. Poitiers (1987).

Quinson, R. INSA. Lyon (1995).

Rafi, O. Poitiers (1982).

\section{Physical Aging of Amorphous Polymers}

Introduction à la thermodynamique des processus irréversibles [Introduction to the Thermodynamics of Irreversible Processes], vol. I. Prigogine. Dunod, Paris, 1968.

The Structure of Non-crystalline Materials. P.M. Gaskell (ed.). London, 1977.

Physical Aging in Amorphous Polymers and Other Materials. L.C.E. Struik (ed.). Elsevier, Amsterdam, 1978.

Basic Feature of the Glassy State. J. Colmenero and A. Alegria (eds.). World Science,

Singapore. 1990

Baur, H. Rheologica Acta 28 (1989): 333.

Curro, J.G. , R.R. Lagasse and R. Simha . Macromolecules 15 (1982): 1621.

Hodge, L.M. . Macromolecules 20 (1987): 2897.

Kovacs, A.J. Adv. Polym. Sci. 3 (1963): 394.

Kovacs, A.J. , J.J. Aklonis , J.M. Hutchinson and A.R. Ramos . J. Polym. Sci-Polym. Phys. ed. 17 (1979): 1907.

Moynihan, C.T. , A.J. Esteal , M.A. Deboit and J.J. Tucker . J. Amer. Ceram. Soc. 59(1976): 12. Narayanaswamy, O.S. J. Amer. Ceram. Soc. 54 (1971): 491.

Perez, J. Polymer 29 (1988): 483.

Perez, J. , Sol. State Lonics 39 (1990): 69.

Perez, J. , J.Y. Cavaill2 and C. Jourdan . Makromol. Chem.. Makromol. Symp., 20/21 (1988):

417.

Perez, J. , J.Y. Cavaill2, R. Diaz-Calleja, J.L. Gomez Ribelles, M. Monleon Pradas and A.

Ribes Creus. Makromol. Chem. (submitted 1991).

Robertson, R.E. , R. Simha and J.G. Curro . Macromolecules 17 (1984): 911.

Scherer, W.J. Non-Cryst. Sol. 123 (1990): 75.

Cavaillé, J.Y. Lyon (1987).

Cunat, C. Nancy (1985).

Bouroukba, M. Nancy (1989). 


\section{Glass Transition}

Les verres et l'état vitreux [The Glasses and the Glassy State]. J. Zarzycky (ed.). Masson,

Paris, 1982.

Glass Transition and the Nature of the Glassy State. M. Goldstein and R. Simha (eds.). New

York Acad. Sci.. New York, 1976.

Relaxations in Complex Systems. K.L. Ngai and G.B. Wright (eds.). Nav. Res. Lab.,

Washington, 1984.

Liquid and Amorphous Metals, Lam 6. W. Glaser , F. Hensel and E. Luscher (eds.). Oldenbourg Verlag. Munich, 1987.

Adam, G. and J.H. Gibbs , J. Chem. Phys. 43 (1965): 139.

Angell, C.A. J. Phys. Chem. Sol. 49 (1988): 863.

Angell, C.A. and G. Wong , J. Chem. Phys. 53 (1970): 2053.

Bengtzelius, W.W. Gotze and A. Sjolander . J. Phys. C17 (1984): 5915.

Chen, H.S. J. Non-Cryst. Sol. 46 (1981): 289.

Cohen, M.H. and G.S. Grest . Phys. Rev. B20 (1979): 1077.

Cyrot, M. J. Phys. C8-41 (1980): 107.

Davies. R.O. and G.O. Jones . Adv. Phys. 28 (1954): 370.

Fischer, E.W. and M. Dettenmaier . Non-Cryst. Sol. 31 (1978): 181.

Gibbs, J.H. and E.A. Di Marzio . J. Chem. Phys. 28 (1958): 373.

Hicter, P. and P. Desré . Les amorphes méalliques [The amorphous metals]. In: Ed. de Phys. B.

Escaig and C. G'sell (eds.). Les Ullis (1983), 1.

Kauzmann, W. Chem. Rev. 43 (1948): 219.

Kovacs, A.J. , J.M. Hutchinson and J.J. Aklonis . In: The Structure of Non-crystalline Materials.

Gas-kell, Taylor \& Francis Ltd., London (1977): 153.

Perez, J. J.Phys. 46, CIO (1985): 427.

Perez, J. , J.Y. Cavaillé and C. Jourdan . Makromol. Chem. Macromol. Symp. 20/21 (1988):

417.

Perez, J. , J.Y. Cavaillé and J. Tatibouet . J. Chem. Phys. 87 (1990): 1923.

Shlesinger, M.F. Ann. Rev. Phys. 39 (1988): 269.

Stillinger. F.H. J. Chem. Phys. 88 (1988): 7818.

Benatmane, A. Lyon (1991).

Bouroukba. M. Nancy (1989).

Cavaillé, J.Y. Lyon (1987).

Cunat, C. Nancy (1985).

Dejean de la Batie, R. Paris (1986).

Etienne, S. Lyon (1985). 